\title{
Strings in the operator formalism
}

\section{Citation}

Alvarez Gaumé, L., C. Gomez, G. Moore, and C. Vafa. 1988. "Strings in the Operator Formalism." Nuclear Physics B 303 (3): 455-521. https://doi.org/10.1016/0550-3213(88)90391-4.

\section{Permanent link}

http://nrs.harvard.edu/urn-3:HUL.InstRepos:41385046

\section{Terms of Use}

This article was downloaded from Harvard University's DASH repository, and is made available under the terms and conditions applicable to Other Posted Material, as set forth at http:// nrs.harvard.edu/urn-3:HUL.InstRepos:dash.current.terms-of-use\#LAA

\section{Share Your Story}

The Harvard community has made this article openly available.

Please share how this access benefits you. Submit a story.

Accessibility 


\title{
Strings in the Operator Formalism
}

\author{
L. Alvarez Gaumé \\ Physics Department \\ Boston University \\ Boston, Ma 02215, USA \\ C. Gomez \\ Theory Division \\ CERN, CH1211 \\ Geneva 23, Switzerland \\ G. Moore \\ Institute for Advanced Study \\ Princeton, NJ 08540, USA \\ C. Vafa \\ Lyman Laboratory of Physics \\ Harvard University \\ Cambridge, Ma 02138, USA
}

We consider string theories in the operator formalism. In particular we develop Polyakov strings completely in the operator language. The operator approach turns out to be economical, self contained and manifestly modular invariant.

$12 / 87$ 


\section{Introduction}

Even though there has been great progress in the last few years on string theory, we seem far from a final formulation of what is meant by string theory. Most of the progress so far has centered around doing perturbative computations on string backgrounds which are classical solutions of string theory, namely the conformal theories. In particular we seem to lack the ability to carry out non-perturbative computations (or even define what is really meant by it). The main questions of predicting the structure of the string vacuum remain as mysterious as ever. Even more importantly, the fundamental principles that lie behind string theory are unkown.

These questions are probably too difficult to answer in the short run, and we may have to get to the answers in stages. We believe that it is important to take some of the clues that string theory has already given us, developing them as best as we can, hoping that these efforts will lead us closer to an understanding of what the theory of string really is.

One of the most important clues we seem to be getting from string theory is that the space of classical solutions to a quantum theory of strings is the space of two dimensional conformal theories. Thus it is important to develop effective means of studying the space
of conformal theories.

Conformal theories have been developed along two different lines. One approach is to use path integrals to define the theory, and the other approach is to use operator techniques. This latter approach may be more general than the former approach, since it is clear that any path integral has an operator content, but not necessarily the other way around. We will follow the operator approach in this paper. The operator formulation is simplest for the sphere and the torus, which has been discussed in detail in the literature. The higher genus case has also been done by sewing lower genus surfaces. The main difficulties in the traditional approach to the operator formalism included the proper treatment of the and the demonstration of modulas invariance, as well as performing explicit computations. It is commonly believed that modular invariance is only manifest in the path integral formulation. This is not necessarily the case, and we will develop the operator approach in a way that maintains the attractive feature of the traditional approach of sewing surfaces, while preserving manifest modular invariance.

It is well known that the energy momentum tensor can be viewed as providing a connection on moduli space. We will see how this is crucial in developing the operator 
formulation of string theory. One of our main aims in this paper is to understand better the connection between the Virasoro algebra (the components of the energy momentum tensor) and moduli space [1]. We can use the Virasoro algebra (and its exponentiated version) to move the states on moduli space. We describe these in the context of the simplest conformal theories, namely the theory of free bosons and fermions, though the setup we use is general enough for an arbitrary conformal theory.

As applications of these methods we give an alternative proof of bosonization com. pletely in the operator language. This will naturally include what may be labeled as chiral bosonization, i.e., the oosonization of left-moving fermions.

Of particular importance for any string theory is the ghost system (fermionic system with spin 2). We will describe the ghost system in detail. Ghosts provide us with the differential forms on moduli space, through which the string measure can be expressed. The ghost number is the same as the degree of the form on the moduli spacel.

As the main application of the operator method we derive the Polyakov measure, as well as the measure for an arbitrary physical scattering amplitude. The advantage of the operator approach is that factorization properties are manifest. Also we never need to know what the vertex operators are for the various physical states. The operator approach we follow could be used to derive the vertex operators, and it is not necessary to know their explicit form for the operator approach. Moreover we deal with all physical amplitudes at once. This is in marked contrast to the path-integral approach where the emission of each physical state requires a different vertex operator. In the operator language there is one global state (for a fixed genus and a fxed number of states) whose projection on different states give different physical scattering amplitudes. This shows that it is this state which is the more fundamental object containing all the information about arbitrary scattering amplitudes at a given order in perturbation theory.

We will discuss the BRST operator, and show how it corresponds to the $d$ operator acting on forms on the moduli space. This is distinct from, but in harmony with, BRST cohomology where the BRST operator is defined as the $d$ operator on the Hilbert space of matter combined with ghosts. Using the BRST operator we will show that the spurious physical states give rise to total derivatives of globally well defined forms on moduli space, and thus they 'formally' decouple after integration on moduli space (modulo contributions from the boundaries). This is well known from the path-integral approach and here is rederived purely in the operator language.

1 This is similar to what happens in string field theory. 
This paper is organized as follows: In section 2 we review the work done on the operator approach to string theory. This will include the recent works as well as the old approach (sewing). This will involve obtaining higher genus Riemann surfaces by sewing lower genus surfaces and the corresponding operator representation. We will review in detail the construction of the operator formulation on an arbitrary genus Riemann surface for free fermions (of arbitrary spin) from several different viewpoints, as well as that of free bosons (of spin zero). The case of free scalars is of particular importance for Polyakov strings (or its toroidal compactifications). In section 3 we develop the operator approach for the ghost system on an arbitrary genus surface. This is one of the key ingredients in understanding how measures on moduli space emerge in an operator language. In section 4 we describe in detail the action of the Virasoro algebra on the moduli space. Section 5 describes the Polyakov string and the measure for an arbitrary physical amplitude. In section 6 we discuss some generalizations. And finally in section 7 we present our conclusions.

In addition we have two appendices: In appendix $A$ we discuss Grassmanians and bosonization formulae. This section is not along the main line of development of this paper, but we include it here because it completes the proof for bosonization in a purely operatorial setup. In appendix B we discuss the Weierstrass gap theorem which is used in the text.

We have been influenced by a large number of works. Even though it is not possible to give proper reference to every relevant work, we try to point out some key references: This work was initiated as the relation between free boson and fermion theories in two dimensions and KP hierarchy were understood (see sec. 2 for details and references). The conformal approach has been formulated in [2]. The connection of conformal theories to string theory has been deacribed in [3]. This connection was further developed in [4]. Also the formulation of physical amplitudes as measures on moduli space of punctured surfaces and the importance of quasi-conformal deformations has been previously suggested in 5 :

\section{Review}

In this section we review some of the recent works on developing an operator formalism for string theory. These works were motivated by some simple systems (like free fermions and bosons). Even though these simple examples are interesting in their own right, they 
do not constitute the most general conformal theory. However they serve as good examples and using them we get a feeling for the general structure of conformal theories.

The general properties of conformal field theories on the complex plane ( the wick rotated version of two dimensional Minkowski space) have been studied in [2][3], and powerful results were obtained using operator methods. What one would like to do, is to obtain a formulation oi conformal theories on higher genus surfaces which keeps the power of the operator formalism on the plane and simultaneously incorporates all the geometrical features characteristic of surfaces with non-trivial topology.

First we recall the connection between the path-integral approach and operator formulation in a two dimensional context. Consider a Riemann surface $\Sigma$ with $n$ parametrized boundaries. Consider a quantum field theory with fields collectively denoted by $\phi$. If we fix the boundary conditions of $\phi$ on the $n$ boundaries, which are $n$ distinct circles labeled by angles $\theta_{i}$, the result of the path-integral is a number. This implies that the path-integral gives us a functional $\Phi$ of the field configurations $\Phi\left(\phi\left(\theta_{1}\right), \ldots, \phi\left(\theta_{n}\right)\right)$. The Hilbert space $*$ of the path-integral is defined to be the functionals of $\phi(\theta)$. This means that the pathintegral on a Riemann surface with $n$ boundaries gives a state in $n$-fold tensor product of the Hilbert space, i.e.,

$$
\Phi \in X^{\otimes n}
$$

We can obtain a closed Riemann surface without boundaries by filling in the boundaries by attaching disks. This means that we simply attach a unit disc to each of the boundaries by identifying the points on the boundaries using the standard parameterization of the boundary of the disc. We label the coordinate of the discs by $t_{i}$. So we see that we can associate to each Riemann surface with $n$ parametrized boundaries, a Riemann surface with $n$ punctures (the centers of the discs) and coordinates $t_{i}$ around the punctures with $\left|t_{i}\right|=1$ corresponding to the $i$-th boundary. The reason for wanting to consider punctured surfaces rather than surfaces with boundaries is that for scattering amplitudes we should imagine a semi-infinite tube attached to each of the boundaries of the surface, representing a particle propagating freely from the infinite past, or to the infinite future. A semi-infinite cylinder is conformally equivalent to the punctured unit disc. And so physical processes involve considering Riemann surfaces with punctures where we imagine the incoming or outgoing particles are placed at the punctures. Scattering amplitudes will be expressed as measures integrated over the space of moduli of punctured Riemann surfaces.

To illustrate some of the basic features of the operator formulation we will now consider some of the simplest of all conformal theories namely the theory of free fermions 
(of spin j) and free bosons (of spin 0). We shall review some of the recent works $[6][7][8][9][10][11][12][13][14]$. Throughout this paper we assume that the reader is familiar with some of the analytic properties of Riemann surfaces [15].

We first consider the theory of free Weyl fermions of spin 1/2 on the Riemann surface. In this section we concentrate on the physical aspects of the operator formulation. We start with a general description of Bogoliubov transformations ${ }^{2}$. Next we show how the Bogoliubov transformations arise upon considering fermions propagating on Riemann surfaces. We connect the operator formalism with the functional integral formulation on a Riemann surface by using holomorphic symmetries of the path-integral. Using this approach we will also develop the operator formulation for scalar fields on higher genus surfaces.

Consider a spin $1 / 2$ Weyl fermion on the complex plane. This could be viewed as conformally equivalent to the propagation of free fermions on a cylinder. Let $z=0,($ resp. $z=\infty)$ be the points corresponding to $\tau=-\infty,($ resp. $\tau=\infty)$ where $z=e^{\tau+i \theta}$ where $(r, \theta)$ label the world sheet coordinates of (euclidean) time and space respectively. The free fermion operators are represented by the expansions

$$
\begin{aligned}
c(z) d z^{1 / 2} & =\sum_{n \in Z+1 / 2} c_{n} z^{n-1 / 2} d z^{1 / 2} \\
b(z) d z^{1 / 2} & =\sum_{n \in z+1 / 2} b_{n} z^{n-1 / 2} d z^{1 / 2} .
\end{aligned}
$$

We have explicitly exhibited the factors $d z^{1 / 2}$ to make clear that both $b(z)$ and $c(z)$ transform as spinor fields. The sums in (2.1),(2.2) indicate that we are considering fermions in the Neveu-Schwarz sector. Since we will work throughout with euclidean field theory, we do not take $b(z)$ to be the hermitian conjugate of $c(z)$. The vacuum is defined by

$$
c_{n}|0\rangle=b_{n}|0\rangle=0 \quad n>0
$$

and the canonical anticommutation relations are

$$
\begin{gathered}
\left|c_{n}, b_{m}\right|_{+}=\delta_{n+m .0} \\
\left.\mid c_{n}, c_{m}\right]_{+}=\left|b_{n}, b_{m}\right|_{+}=0
\end{gathered}
$$

2 We comment on the geometrical interpretation of the Bogoliubov transformations using general properties of infinite grassmannians in the appendix $\mathbf{A}[16]$. 
The functions $1, z, z^{2} \ldots$ are the negative energy states, and $z^{-1}, z^{-2}, \ldots$ are the positive energy states. The standard Dirac vacuum is obtained by filling the $b$ and $c$ negative energy states. The two point function is

$$
\langle 0|c(z) b(w)| 0\rangle=\frac{-1}{z-w}
$$

there is also a conserved fermion number current

$$
\begin{gathered}
j(z)=: c(z) b(z):=\sum_{n \in Z} j_{n} z^{n-1} \\
{\left[j_{n}, j_{m}\right]=\delta_{n+m, 0}}
\end{gathered}
$$

and the normal ordering is defined with respect to the Dirac vacuum $|0\rangle$. Corresponding to the fermion number current we can define a fermion number charge $Q=\frac{1}{2 \pi i} \oint j(z) d z$. The field $c(z)$ increases the charge of any state by one unit, while the field $b(z)$ decreases it by one unit.

We can construct a representation of the free fermionic Fock space in terms of semiinfinite forms. Let $H$ be the space of wave functions of the $b, c$ system (2.1)(2.2).Thus $H=L^{2}\left(S^{1}\right)$ which is spanned by $\left\{z^{n}, n \in Z\right\}$. The inner product in $H$ is given by

$$
\left\langle z^{n} \mid z^{m}\right\rangle=\frac{1}{2 \pi i} \oint_{S^{2}} z^{-n} z^{m} \frac{d z}{z}=\delta_{n, m}
$$

where the contour integral is taken around the origin in the complex plane. The dual wave functions $\theta_{n}$ are defined by

$$
\theta_{n}\left(z^{-m}\right)=\delta_{n, m}
$$

we can write the vacuum $|0\rangle$ as

$$
|0\rangle=\theta_{0} \wedge \theta_{1} \wedge \theta_{2} \ldots
$$

then

$$
b_{n+1 / 2}=\theta_{n} \wedge
$$

and 


$$
c_{n+1 / 2} w_{0} \wedge w_{1} \ldots=\sum_{k>0}(-1)^{k} w_{k}\left(z^{n+1}\right) w_{0} \wedge \ldots w_{k-1} \wedge w_{k+1} \ldots
$$

where the $w$ 's are linear forms on $H$.

A basis for the free Fock space is the familiar set of states with a definite number of particles and antiparticles:

$$
\left|n_{1} \ldots n_{k} ; m_{1} \ldots m_{l}\right\rangle=c_{-n_{1}} \ldots c_{-n_{k}} b_{-m_{1}} \ldots b_{-m_{l}}|0\rangle
$$

these states may also be described in a first quantized language. In $(2.11), 0\rangle$ is constructed by filling the states in $H_{+}=\left\{z^{-n} ; n \geq 0\right\}$. We can construct the multiparticle states (2.14) by filling some subset of wave functions in $H$. For example the state $b_{-1 / 2}|0\rangle$ is obtained by filling the states $z^{1}, 1, z^{-1}, z^{-2}, \ldots$ which represents a particle state, and $c_{-1 / 2}|0\rangle$ is obtained by filling the wave functions $z^{-1}, z^{-2}, \ldots$. This is a hole in (2.11), or an antiparticle. The states in the fermionic Fock space with a finite number of particles and antiparticles can be described by choosing a sequence of integers $n_{1}<\ldots<n_{k} ; m_{1}<\ldots<m_{l}$. The first $k$ of them represent particle states, and the last $l$ the hole or antiparticle states. The subspace of $H$ associated to this sequence of integers is $H_{S}=\left\{z^{n_{2}}, \ldots z^{n_{4}}, 1, z^{-1}, \ldots z^{-m_{1}+1}, z^{-m_{1}-1}, \ldots, z^{-m_{2}+1}, z^{-m_{2}-1}, \ldots\right\}$. More generally we can consider subspaces $W$ which are the span of elements of the form

$$
w_{n}=z^{-n}+\sum B_{n m} z^{m-1}
$$

where we impose suitable growth conditions on the $B_{n m}{ }^{3}$. We can construct a set of wave functions in the dual Hilbert space using the correspondence given in (2.10)

$$
\Omega_{n}=\theta_{n}+\sum_{m} B_{n m} \theta_{-m+1}
$$

from which we make a Fock space state out of the filled states:

$$
|B(W)\rangle=\Omega_{n_{1}} \wedge \Omega_{n_{2}} \wedge \ldots
$$

Using the semi-infinite wedge representation $(2.12),(2.13)$ the state $(2.17)$ can be equivalently.written as

3 Technically the projection of $W$ to $H_{+}$must be Fredholm, which is the mathematical

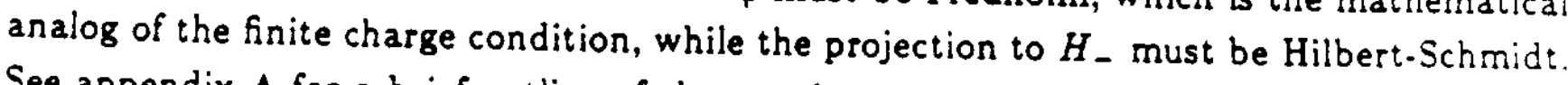
See appendix $\mathbf{A}$ for a brief outline of these technical details. 


$$
\begin{gathered}
\left.|B(W)\rangle=\exp \left(-\sum_{n, m>0} B_{n m} c_{-n+1 / 2} b_{-m+1 / 2}\right): 0\right\rangle= \\
\exp \left(-\frac{1}{(2 \pi i)^{2}} \oint_{P} \oint_{P} B(z, w) c(z) b(w) d z d w\right)|0\rangle=g(B)|0\rangle
\end{gathered}
$$

with

$$
B(z, w)=\sum_{n, m>0} B_{n m} z^{n} w^{m}
$$

this is a Bogoliubov transformation of the Dirac vacuum (2.11).

We should note that all the above has a natural generalization. The fermion current (2.7) defines a natural inner product between $b$ and $c$ wave functions. This is true not only for spinors, but it is also true for more general systems [3] where b has spin $j$, and $c$ has spin $1-j$. Geometrically $b(z)$ behaves like a j-differential, and $c(z)$ as a 1 -j-differential under holomorphic reparametrizations. The scalar product between a b-wave function $w(z)$ and a c-wave function $w^{\prime}(z)$ is then

$$
\left\langle w \mid w^{\prime}\right\rangle=\frac{1}{2 \pi i} \oint_{p} w(z) w^{\prime}(z) d z
$$

the integrand in $(2.21)$ is a $(1,0)$ form, and the integral is conformally invariant. For chira! systems this is the natural inner product. It is intimately connected with the notion of Serre duality on Riemann surfaces [17].

Let us consider a disc, on whose boundary we place the Bogoliubov state $|B(W)\rangle$ (see figure 2.1). Using the standard operator techniques the correlation functions of the fieids on the disc can be computed in terms of the two point function

$$
\frac{\langle 0|b(w) c(z)| B(W)\rangle}{\langle 0 \mid B(W)\rangle}=G(z, w ; B)=\left(\frac{1}{z-w}-B(z, w)\right) d z^{1 / 2} d w^{1 / 2}
$$

using Wick's theorem one obtains

$$
\frac{\left\langle 0\left|\prod_{i=1}^{N} b\left(w_{i}\right) c\left(z_{i}\right)\right| B(W)\right\rangle}{\langle 0 \mid B(W)\rangle}=\operatorname{det}_{i, j} G\left(z_{i}, w_{j} ; B\right)
$$

The state (2.18) can be characterized in a different way. Since $\left.|B(W)\rangle=g(B)_{i}^{\prime} 0\right\rangle$, the state $(2.18)$ is annihilated by the operators 


$$
Q_{n}=g(B) b_{n-1 / 2} g(B)^{-1}=\frac{1}{2 \pi i} \oint_{P} b(z) w_{12}(z) d z \quad n>0
$$

and

$$
Q_{n}^{\prime}=g(B) c_{n-1 / 2} g(B)^{-1}=\frac{1}{2 \pi i} \oint_{P} c(z) w_{n}^{\prime}(z) d z
$$

using (2.4) we readily see that

$$
\left[Q_{n}, Q_{m}^{\prime}\right]_{+}=\left[Q_{n}, Q_{m}\right]_{+}=\left[Q_{n}^{\prime}, Q_{m}^{\prime}\right]_{+}=0
$$

which implies that

$$
\oint_{P} w_{n}(z) w_{m}^{\prime}(z) d z=0
$$

this is a duality relation. From $(2.24)(2.25)$, we obtain

$$
\begin{aligned}
& w_{n}=z^{-n}+\sum_{m>0} B_{n m} z^{m-1} \\
& w_{n}^{\prime}=z^{-n}+\sum_{m>0} B_{m n} z^{m-1}
\end{aligned}
$$

where we have used the symmetry of $B_{n m}$.

We can turn things around, and construct the ray $|B(W)\rangle$ in an algebraic way. We consider the operators $Q_{n}, Q_{m}^{\prime}$ defined according to $(2.24)(2.25)$. These provide a maximal set of compatible anticommuting conditions. The ray $|B(W)\rangle$ is defined by

$$
Q_{n}|B(W)\rangle=Q_{m}^{\prime}|B(W)\rangle=0
$$

if we represent the fermionic states as functionals of $c_{-n}, b_{-m} n, m>0$ the vacuum (2.11) is represented by the function 1 , and the annihilation operators become

$$
b_{n}=\frac{\partial}{\partial c_{-n}} \quad c_{n}=\frac{\partial}{\partial b_{-n}}
$$

thus (2.30) becomes a differential equation whose solution is

$$
|B(W)\rangle=\exp \left(-\sum_{n, m>0} B_{n m} c_{-n+1 / 2} b_{-m+1 / 2}\right)|0\rangle
$$


after one uses (2.28). This description makes it manifest that $|B(W)\rangle$ is a Bogoliubov transformation of the standard vacuum because the operators $Q$ consist of linear combinations of creation and annihilation operators with respect to the standard vacuum, and may be viewed as defining the annihilation operators for the new vacuum.

Now consider a Riemann surface with a single puncture $p$ and with a coordinate $z$ in a neighborhood of the puncture (fig. 2.2) vanishing at $p$. We define the subspace $W$ by considering all the meromorphic sections of the spinor bundle (which for simplicity we assume has no holomorphic sections), which are allowed to have poles only at $p$. In this case we can write an explicit formula for $B_{n m}$ by constructing an explicit basis for meromorphic spinor fields which extend holomorphically to the rest of the surface. The Riemann-Roch theorem implies that we can find meromorphic sections of $L$ which are holomorphic off $p$, and with arbitrary order poles at $p$. These sections give a basis of $W$, and they can be constructed explicitly using the Szego kernel or two point function for fermions on $\Sigma$. The Szego kernel for spin $1 / 2$ is

$$
G(t, y)=\frac{\vartheta\left(\begin{array}{l}
\theta \\
\phi
\end{array}\right)\left(\int_{y}^{t} \omega \mid \tau\right)}{\vartheta\left(\begin{array}{l}
\theta \\
\phi
\end{array}\right)(0 \mid \tau) E(t, y)}
$$

where $(\theta, \phi)$ characterize the different choices of the line bundle (determining their relative twists about the $a$ and $b$ cycles). In (2.33) we are taking coordinates $t(p)=$ $y(p)=0 ; E(t, y)$ is the prime form of $\Sigma, \tau$ is the period matrix for the marking chosen, and the argument of the $\vartheta$ function in the numerator involves the integral of the abelian differentials on the surface and is just the Abel map [18]. A basis for $W$ is obtained by taking successive derivatives of (2.33) with respect to $y$ and setting $y=0$

where

$$
\begin{aligned}
w_{n} & =\left.\frac{1}{(n-1) !} \frac{\partial^{n-1}}{\partial y^{n-1}} G(t, y)\right|_{y=0} \\
& =t^{-n}+\sum_{m=1}^{\infty} B_{n m} t^{m-1}
\end{aligned}
$$

$$
B_{n m}=\frac{1}{(n-1) !(m-1) !} \frac{\partial^{m-1}}{\partial t^{m-1}} \frac{\partial^{n-1}}{\partial y^{n-1}}\left(G(t, y)-\frac{1}{(t-y)}\right) .
$$

Consier the ray $|B(W)\rangle$ with these values for $B_{n m}$. To connect this to the theory of fermions on Riemann surfaces we must show how the same ray can be derived from 
a path-integral point of view. In particular, since the ray $|B(W)\rangle$ is characterized by the infinitely many annihilation conditions $(2.30)$ it suffices to show why these operators correspond to conserved charges arising from symmetries of the path-integral. Then the annihilation conditions (2.30) simply translate into statements about the symmetries of the conformal theory. Imagine that we do the functional integral for the $b, c$ system of spin $1 / 2$ on a surface $\Sigma$ minus a disc. The result will give a state on the boundary of the disc. To establish what functional integral has to be computed, let us consider the more general case in fig. 2.3 of a surface $\Sigma$ which is divided into two pieces by a circle $S^{\mathfrak{l}}$. We would like to represent the result of functionally integrating over the whole surface as an overlap integral on the Fock space of the circle of two states $\Psi_{1}[f]$ and $\Psi_{2}[f]$, where $f(z)$ are the field configuration of $c(z)$ on $S^{1}$. In order to make the computations as simple as possible, we use the holomorphic representation of the functional integral [19]. Thus we represent the fermionic wave functionals as functionals of the possible values of either $c$ or $b$ on $S^{1}$. More concretely, to obtain the state associated to performing the functional integral over $\Sigma_{1}$, we impose the boundary condition $\left.c(z)\right|_{s^{1}}=f(z)$, and no boundary condition on $b(z)$. The reason we do this is that in the coherent state formulation of the fermionic functional integral we always work with the hamiltonian formulation of field theory. Here we take $c(z)$ as the coordinates, and $b(z)$ as the canonical conjugate momenta. If we write down the explicit form of the evolution kernel for a system of free fermionic oscillators, there is an extra boundary term in the action which accounts for the flux through $S^{l}$ of the fermionic current. Another way of understanding the origin of the boundary term is to notice that the equation of motion for the $b$ field $\bar{\partial} b=0$ is obtained only if we add the boundary term. This term cancels a similar term with opposite sign from the state associated to $\Sigma_{2}$. These terms are there to match the fermionic current on both sides of $S^{1}$. The state is thus

$$
\Psi(f)=\int_{\left.c\right|_{s 1}=f} d b d c \exp \left(-\int_{\Sigma_{1}} b \bar{\partial} c+c \bar{\partial} b+\oint_{S^{2}} c b\right)
$$

in the Fock space of $S^{l}$, the operator $c(z)$ is represented as multiplication by $c(z)$ and $b(z)$ becomes $\partial / \partial c(z)$. The wave function $(2.37)$ is invariant under shifts of $f(z)$ by sections of $c(z)$ holomorphic in $\Sigma-p$, i.e., $c \rightarrow c+w_{n}$. The resulting symmetry implies

$$
\Psi\left(f+w_{n}\right)=\Psi(f)
$$


since translations of $f$ are implemented by the operator $\oint_{S} w_{n} b(z)=Q_{n}$, this establishes half of (2.30). To show that $Q_{n}^{\prime}$ are also a symmetry of $\Psi$ we can choose the boundary condition which fixes $b$ field at the boundary. It is clear that $\Psi$ is a state in the Hilbert space and it is independent of how we wish to perform the functional integral. This finally establishes that $Q_{n}$ and $Q_{n}^{\prime}$ are indeed conserved charges arising from conformal symmetries of the path-integral, and we recover (2.30) and (2.32) (with $\Psi$ being identified with $|B(W)\rangle)$.

In appendix $A$ we will use this result together with the characterization of bosonic states on a Riemann surface to obtain an algebraic proof of the bosonization formulae for $b, c$ systems on any Riemann surface [20][21][22][23].

The argument just presented works for $b, c$ systems of arbitrary spin $j, 1-j$. When $j>1$, and $g>1$, the Riemann-Roch theorem [17] implies that there are $(2 j-1)(g-1)$ global holomorphic sections of $b(z)$. This is equivalent by the duality relation (2.27) to saying that the holomorphic $1-j$ differentials for $\Sigma-p$ must have poles at $p$ of order higher than $(2 j-1)(g-1)$. Thus there are $(2 j-1)(g-1)$ 'more ' $Q$ ' conditions than $Q$ conditions. The state (2.37) has an excess charge of $(2 j-1)(g-1)$ with respect to the standard vacuum on the sphere. Consequently, any correlation function $\langle c(z) \ldots b(w) \ldots \mid W\rangle$ will vanish unless there are precisely $(2 j-1)(g-1)$ more $b$ than $c$ field insertions. We will treat the case of spin 2 system in more detail in sec. 3. The other cases will be analogous and should be clear from the above treatment combined with the discussion in section 3 .

The idea of the conserved charges generalizes to arbitrary conformal theories where there are fields $\phi$ of integer or half integer spin which satisfy the equation $\bar{\partial} \phi=0$. For $\phi$ to have a well defined spin, we must require it to be a primary field [2]. In such cases we consider the meromorphic sections $\eta$ of an appropriate bundle so that $\eta \phi$ is a $(1,0)$ form where we consier all sections $\eta$ which have no poles except at the puncture. Since $\eta \phi$ is a one form it can be integrated around the puncture,

$$
Q=\oint \eta \phi
$$

and the resulting operator should be a conserved charge and annihilate the state. The reason is that we can deform the contour without encountering any poles, and pull off the contour from the other side of the surface. In a general conformal theory these would give some conditions on the states, but they are not sufficient in general to determine the state, as was the case here. 
Now we consider a free scalar field on a Riemann surface. There are two cases of interest. Single-valued and multi-valued fields (the fields being angle variables). In string language they correspond respectively to coordinates of uncompactified (straight line) or compactified space (circle), where strings are propagating. The first case is simpler, and it will be treated in this section. The multivalued case will be considered in appendix A in connection with bosonization. As in the previous case, we want to construct a bosonic wave functional $\Psi[f]$ associated to the surface $\Sigma_{1}$ and the scalar field. We obtain a functional integral representation for this wave functional using once again the hamiltonian formulation of the path integral. As in the fermionic case, we find a boundary term which accounts for the normal derivative of the scalar field on the boundary circle

$$
\Psi[f]=\int_{\left.X\right|_{s^{1}=f}} d X \exp \left(-\frac{1}{8 \pi} \int_{\Sigma_{1}} d X \wedge * d X+\frac{1}{8 \pi} \oint_{S^{3}} X * d X\right)
$$

where $*$ is the duality operation on forms $* d z=-i d z, * d \bar{z}=i d \bar{z}$. Since $X$ is a real scalar field, we can uniquely extend $f$ to a smooth harmonic function on $\Sigma_{1}$ which we continue to call $f$. Any correlations involving the field $X$ on the boundary could be computed by replacing $X$ (or its derivatives) using the function $f$ (or its derivatives). It is in this sense that one has to understand the normal derivative on the boundary appearing in (2.39). This wave functional is invariant under shifts of $f$ by any harmonic function on $\Sigma_{1}$. The proof uses Green's identity for harmonic functions

$$
\oint_{S^{1}}\left(\phi_{1} * d \phi_{2}-\phi_{2} * d \phi_{1}\right)=0
$$

thus we can construct the state (2.39) by requiring that it be annihilated by the corresponding conserved charges. The complication appears when we try to find explicit expressions for these harmonic functions. The simplest solutions one can think of are the real and imaginary parts of a holomorphic function on $\Sigma-p$ allowed to have poles at $p$. By the Weierstrass gap theorem ([17], see also appendix $B$ ) we know that for a generic point $p$ the order of the pole of a holomorphic function on $\Sigma-p$ must be bigger than $g$. Thus we need $g$ extra conditions to determine completely the state (2.39). It turns out that we can fill the gaps by considering harmonic functions which have both holomorphic and antiholomorphic pieces. We can start by constructing a function which is holomorphic and has a pole of order less than $g$. As discussed, this function cannot be well defined. But we can construct such a function if we allow multivaluedness as we go around the cycles. Since solutions to the equations of motion are harmonic functions we can add to 
any holomorphic function with poles at $p$ some antiholomorphic piece, so that we obtain a single valued function without the restrictions imposed by the gap theorem. Explicitly, if $\omega_{i}, \bar{\omega}_{i}$ are the abelian differentials and we introduce the differentials

$$
\eta_{n}(t)-A_{n}(I m \tau)^{-1}(\omega-\bar{\omega})
$$

where

$$
\omega_{i}(t)=\sum_{n=1}^{\infty} A_{i n} t^{n-1} d t
$$

and

$$
\eta_{n}(t)=-\left.\frac{1}{(n-1) !} \frac{\partial}{\partial t} \frac{\partial^{n}}{\partial y^{n}} \ln E(t, y)\right|_{y=0}
$$

it is easy to check using the properties of the prime form [18] that

$$
X_{n}(t)=\int^{t}\left(\eta_{n}(t)-A_{n}(I m \tau)^{-1}(\omega-\bar{\omega})\right)
$$

is a single valued harmonic function on $\Sigma-p$. Expanding in power series of $t$

$$
X_{n}(t)=X_{n}^{h}+X_{n}^{a}
$$

with

$$
\begin{gathered}
X_{n}^{h}=t^{-n}-\sum_{n=1}^{\infty}\left(2 Q_{n m} \frac{t^{m}}{m}+\pi A_{n}(I m \tau)^{-1} A_{m} \frac{t^{m}}{m}\right) \\
X_{n}^{a}=\pi A_{n}(\operatorname{Im})^{-1} \bar{A}_{m} \frac{\bar{t}^{m}}{m}
\end{gathered}
$$

and the coefficients $Q_{n m}$ are given by the multiple logarithmic derivatives of the prime form

$$
Q_{n m}=\left.\frac{1}{2(n-1) !(m-1) !} \frac{\partial^{n}}{\partial t^{n}} \frac{\partial^{m}}{\partial y^{m}} \log \frac{E(t, y)}{(t-y)}\right|_{t=y=0}
$$

the oscillator expansion of the scalar field $X(t)$ is

$$
X(t)=q+i p \ln t+i p \ln \bar{t}+i \sum_{n \neq 0}\left(\frac{t^{n}}{n} j_{n}+c . c .\right)
$$


and the non-vanishing commutation relations are

$$
[q, p]=i,\left[j_{n}, j_{m}\right]=n \delta_{n+m, 0}, \quad\left[\bar{j}_{n}, \bar{j}_{m}\right]=\delta_{n+m, 0}
$$

(this means that we can think of $j_{n}$ and $j_{-n}$ as annihilation and creation operators respectively, with $n>0$ ). The operators $j_{n}$ generate a $U(1)$-current algebra, an infinite dimensional Heisenberg algebra, and they can be written in the Schrödinger representation

$$
\begin{array}{ll}
j_{n}=-i \frac{\partial}{\partial x_{n}} & j_{-n}=i n x_{n} \\
\bar{j}_{n}=-i \frac{\partial}{\partial \bar{x}_{n}} & \bar{j}_{-n}=i n \bar{x}_{n}
\end{array}
$$

since the equation of motion for the field $X$ is

$$
\partial \bar{\partial} X=0
$$

the conserved charge obtained by the shift $X \rightarrow X+\phi$ with $\phi$ harmonic in $\Sigma-p$ is

$$
Q(\phi)=i \oint_{S^{2}}(\phi * d X-X * d \phi)
$$

in terms of local currents, this means that

$$
j(\phi)=\partial X \phi^{h}-\bar{\partial} X \phi^{\circ}
$$

is conserved. Using the commutation relations (2.50), and Green's theorem $(2.40)$ it can be shown that the commutator between any two charges $Q\left(\phi_{1}\right), Q\left(\phi_{2}\right)$ vanishes. Substituting (2.44) in (2.54) one obtains the explicit form of the conserved charges

$$
Q_{n}=j_{n}-\sum_{n=1}^{\infty}\left(2 Q_{n m}+\pi A_{n}(I m \tau)^{-1} A_{m}\right) \frac{j-m}{m}+\pi A_{n}(I m \tau)^{-1} \bar{A}_{m} \frac{\bar{j}_{-m}}{m}
$$

and the differential conditions for the wave functional (2.39) are

$$
Q_{n}|\phi\rangle=\bar{Q}_{n}|\phi\rangle=0
$$

whose complete solution is 
where

$$
|\phi\rangle=C \exp \left((x, \bar{x}) M\left(\frac{x}{\bar{x}}\right)\right)
$$

$$
M=\left(\begin{array}{cc}
Q_{n m}+\frac{\pi}{2} A_{n}(I m \tau)^{-1} \dot{A}_{m} & -\frac{\pi}{2} A_{n}(I m \tau)^{-1} \bar{A}_{m} \\
-\frac{\pi}{2} \bar{A}_{n}(I m \tau)^{-1} A_{m} & \bar{Q}_{n m}+\frac{\pi}{2} \bar{A}_{n}(i m \tau)^{-1} \bar{A}_{m}
\end{array}\right)
$$

the overall normalization constant $C$ is a function of the moduli parameters of the surface (and the actual metric chosen for the surface, if the conformal anomaly does not vanish), and it will be shown in sec. 5 that it is related to the determinant of the Laplacian on $\Sigma$. Notice that the state $|\phi\rangle$ has zero momentum. This is because a constant shift of $X$ is a symmetry of the functional integral, and its conserved charge is the momentum operator $p$.

The next case of interest in bosonization as well as in toroidal compactifications of strings is the case of multivalued scalars which are defined up to addition by a constant. Since the explicit form of the state obtained for these fields on any surface will only be needed in the proof of bosonization in appendix $A$, we defer to that section the explicit construction and study of the peculiarities of this system.

So far we have considered the case of a Riemann surface with one puncture. Nothing prevents us from considering the case of Riemann surfaces with more punctures ${ }^{4}$. In fact these would be of interest in the computations of scattering amplitudes, where each puncture will be associated to an incoming or outgoing particle. So we consider a Riemann surface with $n$ punctures, and coordinates $z_{i}$ chosen in a neighborhood of the punctures (fig. 2.4). If we cut out discs with radius $\left|z_{i}\right|=1$, we get a Riemann surface with $n$ boundaries. We are assuming that the discs do not intersect. Below we will comment on the more general case. The result of the path-integral on the Riemann surface with $n$ boundaries is to give a state on the $n$-fold tensor product of the Hilbert space.

Let us concentrate on the theory of free bosons of spin zero. We will construct the corresponding ray by the conserved charge method. In the case of the single puncture we saw that we have two infinite sets of conserved charges corresponding to harmonic

1 These have been considered for the case of sphere with $n$ punctures from the same viewpoint in $|24|$. 
functions which are allowed to have poles at the puncture. Consider a harmonic function $f$ which has a pole only at the puncture $z_{1}=0$. Near this puncture we have

$$
f=z_{1}^{-m}+\sum_{k \geq 0} A_{m k}^{1} z_{1}^{k}+B_{m k}^{1} \bar{z}_{1}^{k}
$$

and it has some power series expansion near the other punctures:

$$
f=\sum_{k \geq 0} A_{m k}^{i} z_{i}^{k}+B_{m k}^{i} \bar{z}_{i}^{k}
$$

When we consider shifting the path-integral to obtain the conserved charges we see that this harmonic shift of the field $X$ gives a sum of $n$ operators, one for each Hilbert space. For $f$ we obtain the conserved charge

$$
Q(f)=\frac{1}{2 \pi i} \sum_{i} \oint_{x_{i}=0} f_{h}\left(z_{i}\right) \partial X-f_{a}\left(\bar{z}_{i}\right) \bar{\partial} X
$$

where $f_{h}$ and $f_{a}$ denote the holomorphic and antiholomorphic parts of $f$ respectively. If we label the basic variables of Hilbert space of the punctures with $\tau_{k}^{i}$ where $i$ labels the Hilbert space, the above conserved charge translates to the differential equation

$$
\frac{\partial}{\partial x_{m}^{1}}-\sum_{k, i} A_{m k}^{i} x_{k}^{i}+B_{m k}^{i} \vec{x}_{k}^{i}
$$

For each puncture and each $m$ we can find such an $f$ (and similarly for the complex conjugate singularity). In this way we find a complete set of equations which could have at most one solution. That a solution exists follows from the fact that the corresponding charges commute:

$$
\left.[Q(f), Q(g)]=\frac{1}{2 \pi i} \sum \oint_{z_{i}=0} \partial\left(f_{h}\left(z_{i}\right)\right) g_{h}\left(z_{i}\right)-\bar{\partial}\left(f_{a}\left(\bar{z}_{i}\right)\right) g_{a}\left(\bar{z}_{i}\right)\right)=0
$$

(using equation (2.40)). The solution can be written in terms of the Green's functions, as for the once punctured case. Let

$$
G\left(z_{i}, w_{j}\right)=-2 \pi \operatorname{Im}\left(\int_{z_{i}}^{w_{j}} \omega\right)(\operatorname{Im} \tau)^{-1} \operatorname{Im}\left(\int_{z_{i}}^{w} \omega\right)-\ln E\left(z_{i}, w_{j}\right)-\ln E\left(\bar{z}_{i}, \bar{w}_{j}\right)
$$

where $\omega$ denotes the $g$ holomorphic one forms, and $\tau$ the period matrix, and $E\left(z_{i}, w_{j}\right)$ denotes the prime form (see $[18])^{5}$. In addition we need the regularized Green's function, which subtracts off leading singularity if $z$ and $w$ are in the same patch:

$$
g\left(z_{i}, w_{j}\right)=G\left(z_{i}, w_{j}\right)+\delta_{i j}\left(\ln \left(z_{i}-w_{i}\right)+\ln \left(\bar{z}_{i}-\bar{w}_{i}\right)\right)
$$

5 Actually the prime form is a differential form so the above formula for the Green's function has extra terms in it. These don't matter in the present treatment. 
We can split $g\left(z_{i}, w_{j}\right)$ to four pieces depending on whether the contributions are holomorphic or antiholomorphic in each of the two variables which we denote by $g_{h h}, g_{h a}, g_{a h}, g_{a a}$. We furthermore consider the matrix $M\left(z_{i}, w_{j}\right)$ to be the $2 \times 2$ matrix whose entries are the four pieces of $g$. Then the unique ray corresponding the Riemann surface with $n$ discs cut out for the bosonic system is

$$
|\phi\rangle=\exp \left(\frac{1}{2}\left(\frac{1}{2 \pi i}\right)^{2} \sum_{i, j} \oint \oint_{z_{i}, w_{j}=0}\left(\partial X\left(z_{i}\right) \bar{\partial} X\left(\bar{z}_{i}\right)\right) M\left(z_{i}, w_{j}\right)\left(\begin{array}{l}
\partial X\left(w_{j}\right) \\
\bar{\partial} X\left(\bar{w}_{j}\right)
\end{array}\right)\right)|0\rangle
$$

There are also the constant modes which give rise to momentum conservation which has been left out of the above equation. Including that replaces the $|0\rangle$ in the above by

$$
\int \prod d^{d} p_{i} \delta\left(\sum p_{i}\right)\left|p_{1}, \ldots, p_{n}\right\rangle
$$

This concludes our derivation of the ray.

Note that in the above derivation, even though in order to give a path-integral derivation we needed to assume that the discs did not overlap, the actual steps we went through in obtaining the ray did not make any reference to this fact. All we needed were certain punctures, and coordinates chosen at the punctures (In some sense, the state is rather like a germ of an analytic function (25!). The ray we obtained makes perfect sense even if the discs overlap, but then it loses its correspondence with the space of Riemann surfaces with discs cut out ${ }^{\sigma}$. This means that the space we are considering, namely the space of Riemann surfaces of a given genus $g$ with $n$ punctures and $n$ coordinates in the neighborhood of the puncture, which we shall denote by $P(g, n)$, is somewhat more general than the space of Riemann surfaces with parametrized boundaries. As we mentioned earlier any Riemann surface with parametrized boundary, can be used to give a point in $P(g, n)$ by attaching to the boundaries unit discs with the parameterization at the boundary matching the standard parameterization of the boundary of the disc.

We note some basic facts about the rays that we get. The ray corresponding to the sphere with one puncture at $z=0$ with coordinate $z$ is the vacuum $|0\rangle$. This follows easily by noting that the corresponding Bogoliubov transformation is one. For the sphere with two punctures, one at 0 the other at $\infty$, with coordinates $z$ and $1 / z$ respectively, the corresponding state for the bosonic Hilbert space is easily seen to be

$$
\left|S_{12}\right\rangle=\int d^{d} p \exp \left(a_{n}^{1 \dagger} a_{n}^{2 \dagger}+\bar{a}_{n}^{1 \dagger} \bar{a}_{n}^{2 \dagger}\right)|p\rangle_{1} \otimes|-p\rangle_{2}=\sum|n\rangle \otimes|n\rangle
$$

\footnotetext{
6 In such a case we do not expect the ray to have a finite norm.
} 
where the sum runs over a complete orthonormal basis (together with a momentum integral) where $a_{n}^{\dagger}=i \sqrt{n} x_{n}$. In case we were considering an indefinite metric structure for bosons, there would be a factor $(-1)$ for each term in the above sum which corresponds to a state with negative norm $\langle n \mid n\rangle=-1$.

Now we review the discussion in [13] of the sewing of two Riemann surfaces, and how that is realized at the level of Hilbert spaces. Consider two Riemann surfaces with punctures and coordinates, labeled by $P \in P\left(g_{1}, n_{1}\right)$ and $Q \in P\left(g_{2}, n_{2}\right)$. Consider one puncture on each surface with coordinates $t_{i}$ and $t_{j}$ respectively. We can glue the two surfaces by identifying the coordinates according to $t_{i}=1 / t_{j}$ (this is plumbing fixture with parameter one; more generally we can consider identifying $t_{i}=q / t_{j}$ with plumbing parameter $q$ ). Identification means that we cut out an annulus around each puncture, such that the inner radius of each annulus corresponds to the outer radius of the other under the above coordinate identification. In terms of circle identifications we can think of this operation as gluing the two Riemann surfaces with boundaries, obtained by cutting out unit discs around the punctures $i$ and $j$, by identifying the circle at $\left|t_{i}\right|=1$ with the one at $\left|t_{j}\right|=1$, according to $t_{i}=1 / t_{j}$. Note that we can equivalently describe this operation by identifying a circle denoted by $\left|t_{i}\right|=a$ with the circle denoted by $\left|t_{j}\right|=1 / a$. We assume that no other punctures are in the regions of identification. We obtain in this way a Riemann surface of genus $g=g_{1}+g_{2}$ and $n=n_{1}+n_{2}-2$ punctures labeled by $R \in P(g, n)$. This operation is denoted by $R=P_{i} \infty ; Q$ (see fig. 2.5).

Similarly, if we consider a single Riemann surface, with more than one puncture, labeled by $P$ we can obtain a Riemann surface in one higher genus, with two fewer punctures, by identifying two of the coordinate patches $i$ and $j$ as described above. The resulting operation is denoted by $P 8_{j}^{i}$ (see fig. 2.5).

Now we come to the realization of these operations in the Hilbert space. We consider the case of the $\infty$ operation. The other operation can be done analogously. Let $|\phi\rangle_{P}$ and $|\phi\rangle_{Q}$ denote the rays corresponding to $P$ and $Q$ respectively. As discussed we can view these as statea which we get at the boundaries of the Riemann surfaces where we have cut out a unit disk centered at the punctures. We suppose for simplicity that the discs do not overlap. Joining the circle corresponding to $i$-th puncture of $P$ to the $j$-th puncture of $Q$, means identifying the two Hilbert spaces. It is clear that the result of the path-integrat on the surface $R$ could be obtained by first considering the corresponding path-integrals on $P$ and $Q$ and writing the $i$-th Hilbert space of $P$ as a bra state, and the $j$-th Hilbert space as a ket space and then taking the inner product between them. Taking the $i$-th Hilbert 
space and reversing the ket to a bra, requires time reversal operation on the Hilbert space, which is obtained by the appropriate choice of a real basis, which is done as follows:

$$
\sum_{n} i\langle n|\left(i\langle n \mid \phi\rangle_{P}\right)
$$

Now taking the inner product of this state with $|\phi\rangle_{Q}$ by identifying the $i$-th Hilbert space with the $j$-th one, we have

$$
|\phi\rangle_{P, \infty, Q}=\sum_{n}\left(i\langle n \mid \phi\rangle_{P}\right)\left(j\langle n \mid \phi\rangle_{Q}\right)=\left\langle S_{i j}|| \phi\right\rangle_{P}|\phi\rangle_{Q}
$$

where $\left|S_{i j}\right\rangle$ is the state obtained for the sphere with two punctures and coordinates $z$ and $1 / z$, and may be called the sewing state. Geometrically we know that if we had sewed the circle of radius $\left|t_{i}\right|=a$ with the circle $\left|t_{j}\right|=1 / a$, we should get the same circle. It is easy to convince oneself (and we shall establish it in detail in section 4 ) that the corresponding states on the circles are

$$
|\phi\rangle_{P} \rightarrow a^{L_{0}} \bar{a}^{\bar{L}_{0}}|\phi\rangle_{P} \quad|\phi\rangle_{Q} \rightarrow a^{-L_{0}}(\bar{a})^{-\bar{L}_{0}}|\phi\rangle_{Q}
$$

which implies that the result of sewing

$$
|\phi\rangle_{R} \rightarrow \sum_{n} i\left\langle n\left|a^{h_{n}} \bar{a}^{\bar{h}_{n}}\right| \phi\right\rangle_{P_{j}}\left\langle n\left|a^{-h_{n}} \bar{a}^{-\bar{h}_{n}}\right| \phi\right\rangle_{Q}=|\phi\rangle_{R}
$$

is the same (where $h_{n}\left(\bar{h}_{n}\right)$ denote the eigenvalues of $L_{0}\left(\bar{L}_{0}\right)$ of $\left.|n\rangle\right)$, as expected. Now suppose we have a Riemann surface with punctures and coordinates, such that the unit discs determined by the coordinates at the punctures overlap. If one were to sew this Riemann surface to another, one might think that it should not make sense to sew along the boundaries of discs which overlap. However this may not be a problem, because we can rescale one coordinate of the disc and scale in the opposite direction the coordinate of the other Riemann surface in such a way that the resulting sewing does not change as discussed above. This shows another difference between viewing the state on the boundaries of Riemann surface as opposed to viewing the states as a kind of Heisenberg state associated to a Riemann surface with punctures and coordinates where the coordinates determines slices of constant time.

The derivation for the sewing operation we gave for the bosons was based on the path-integral interpretation. We can justify this procedure completely in the operator 
formulation in the following way. For the sake of definiteness we consider the $\infty$ operation, the other operation 8 being analogous. We note that the ray in the operator formulation is completely specified by the conserved charges. So what we have to show is that if some harmonic function gives rise to a conserved charge for $R$, the ray defined by $(2.60)$ as the result of sewing of the corresponding rays on $P$ and $Q$ is annihilated by it. A conserved charge corresponding to a harmonic function $f$ on the surface of $R$, receives contribution from all the boundaries of $R$ and may be written as a linear sum of operators

$$
Q(f)=Q_{1}+\ldots+Q_{i-1}+Q_{j+1}+\ldots+Q_{r}
$$

where labels $1, \ldots, i$ label the punctures on $P$ and $j, \ldots, r$ label punctures on $Q$. We can use coordinates $t_{i}$ and $t_{j}$ as some coordinate patch for $R$, only an annulus of which is part of $R$. Expanding the function $f$ in these coordinate patches gives rise to operators $Q_{i}$ and $Q_{j}$ which satisfy

$$
\left\langle S_{i} j\left(Q_{i}+Q_{j}\right)=0\right.
$$

as follows from the fact that sewing operator is the state associated to a sphere with two punctures and coordinates $z, 1 / z$. We therefore have

$$
\begin{gathered}
Q_{1}+\ldots Q_{i-1}+Q_{j+1}+\ldots+Q_{r}\left\langle S_{i j}|| \phi\right\rangle_{P}|\phi\rangle_{Q}= \\
\left\langle S_{i j}\left|Q_{1}+\ldots+Q_{i-1}-\left(Q_{i}+Q_{j}\right)+Q_{j+1}+\ldots+Q_{r}\right| \phi\right\rangle_{P}|\phi\rangle_{Q}= \\
\left\langle S_{i j}\left(\left(Q_{1}+\ldots+Q_{i}\right)|\phi\rangle_{P}|\phi\rangle_{Q}+|\phi\rangle_{P}\left(Q_{j}+\ldots+Q_{r}\right)|\phi\rangle_{Q}\right)=0\right.
\end{gathered}
$$

where the last equality follows by the definition of the ray at $P$ and $Q$. This completes what was to be shown. The argument which we just went through is very useful in the operator formulation, and we shall use it in the subsequent sections of this paper. It essentially is the statement that symmetries of our conformal theories are preserved by the sewing operation. We shall call the above argument the charge transport argument (CTA).

\section{Ghost System}

In this section we develop the operator approach for the ghost system. We take a spin 2 (quadratic differential) fermion, denoted by $b$ and a spin -1 (vector field) fermion denoted by $c$. We first review the ghost system on the sphere (26)|3]. We will discuss the holomorphic ghosts; the anti-holomorphic ones $\bar{b}$ and $\bar{c}$ are simply the complex conjugate 
version of what we do here. Also in the paper occasionally we will refer to the ghost system by considering only the holomorphic ghosts. It should be easy to decide from the context whether both holomorphic and antiholomorphic ghosts are intended or not. We consider the expansions

$$
b(z)=\sum b_{n} z^{n-2} \quad c(z)=\sum c_{n} z^{n+1}
$$

with the anti-commutation relations

$$
\left.\left[\dot{o}_{n}, c_{m}\right]_{+}=\delta_{n+m, 0} \quad\left[b_{n}, b_{m}\right]_{+}=\therefore i_{n}, c_{m}\right]_{+}=0
$$

We define the ghost vacua $|+\rangle$ and $|-\rangle$ by

$$
\begin{gathered}
b_{n}|-\rangle=0=c_{n}|+\rangle \quad \text { for } n \geq 0 \\
c_{0}|-\rangle=|+\rangle \quad b_{0}|+\rangle=|-\rangle .
\end{gathered}
$$

Furthermore, the Hilbert space inner product is dictated by demanding that $b_{n}^{\dagger}=b_{-n}$ and $c_{n}^{\dagger}=c_{-n}$, and also by

$$
(| \pm\rangle)^{\dagger}=\langle \pm| \quad\langle+\mid-\rangle=1 \quad\langle+\mid+\rangle=\langle-\mid-\rangle=0
$$

The energy momentum tensor is defined by

$$
L_{n}=\sum(n-m) b_{n+m} c_{-m}
$$

with the normal ordering for $L_{0}$ defined so that

$$
L_{0}| \pm\rangle=(-1)| \pm\rangle
$$

It is easy to verify that

$$
\left[L_{n}, L_{m}\right\rfloor=(n-m) L_{n+m}+\frac{-26}{12}\left(n^{3}-n\right) \delta_{n,-m} .
$$

Consider the Riemann sphere, with the standard coordinate $z$ chosen around the origin. We would like to know what state $\left|\phi_{0}\right\rangle$ in the Hilbert space to associate with it. As described in the last section, the path integral is completely characterized up to a constant by the fact that the state is annihilated by the conserved charges. Let $v$ be a holomorphic 
- I-differential on the sphere which is allowed to have poles only at the origin. Then, as described in the last section, we must have

$$
\oint b(z) v(z)\left|\phi_{0}\right\rangle=0
$$

and similarly for a quadratic differential $s$ that is allowed to have poles only at the origin we must have

$$
\oint c(z) s(z)\left|\phi_{0}\right\rangle=0 \text {. }
$$

It is easy to choose a basis for $v$ and $s$ of the form

$$
v=z^{2}, z^{1}, 1, z^{-1}, \ldots \quad s=z^{-4}, z^{-5}, \ldots
$$

(these are found by demanding no poles at $\infty$ ). These give rise to the infinite set of conserved charges

$$
b_{-1}, b_{0}, b_{1}, \ldots \quad c_{2}, c_{3}, c_{4}, \ldots
$$

The conditions (3.7) and (3.8) are easily seen to be uniquely solved (up to an undetermined normalization) by

$$
\left|\phi_{0}\right\rangle=b_{-1}|-\rangle=b_{-1} b_{0}|+\rangle
$$

We will denote the states $\left|\phi_{0}\right\rangle_{0}$ and $\left\langle\phi_{0}\right|$ by $|0\rangle$ and $\langle 0|$ respectively and think of them as the ghost vacua. It is easy to verify that

$$
L_{n}|0\rangle=0 \quad \text { for } \quad n \geq-1
$$

It is very useful to define the ghost number of a state in the Hilbert space. We take $c_{n}$ to change ghost number by $1, b_{n}$ to change ghost number by -1 , and the $| \pm\rangle$ vacua to have $\pm 1 / 2$ ghost number respectively. So the state $|0\rangle$ has ghost number $-3 / 2$ (as does the conjugate state $\langle 0|=\left\langle-\mid b_{1}\right\rangle$. This in particular means that if we want to get a nonzero matrix element for $\langle 0|\ldots| 0\rangle$ we must insert an operator with ghost number 3 (i.e., the number of $c$ fields should be 3 more than the number of $b$ felds). This is simply a result of the index theorem, which for $\partial$ operator acting on 2-differentials on the sphere has index equal to -3 . The ghost number of $|0\rangle$ could also be understood by considering the index of the $\partial$ operator on the sphere with one boundary $(a t|z|=1)$ with a suitable boundary condition. 
Now let us discuss the higher genus case. We consider $g>1$; the case of the torus is left as an exercise to the reader. A fundamental fact which we will use in the following is the Weierstrass gap theorem for $j$-differentials. This is described in appendix B.

Consider a Riemann surface $\Sigma$ of genus $g$ with a single puncture at $p$ and a local parameter $t$ vanishing at $p$. Consider quadratic differentials which are holomorphic on $\Sigma-p$ and are allowed to have poles only at $p$. There are $3 g-3$ quadratic differentials without any poles anywhere. Generically we can choose a basis for them by demanding they vanish at $t=0$ to a given order $0 \leq n<3 g-3$. We represent the corresponding quadratic differential by $\psi_{n+1}$. This means that in the neighborhood of $p$

$$
\psi_{n+1}=t^{n}+\sum_{m=n+1}^{\infty} C_{n m} t^{m}
$$

At certain points (Weierstrass points) on the Riemann surface, there will be some gaps for some $0 \leq n<3 g-3$ for which no $\psi_{n+1}$ exists, and instead there are additional holomorphic quadratic differentials which vanish to a higher order. Again for uniformity we will consider being off these points. It is straightforward to modify our arguments below in such a case.

For each positive integer $n$ it is possible to find a quadratic differential which has a pole of order $n$ at $p$. This section is labeled by $s_{n}$. We have

$$
s_{n}=t^{-n}+\sum_{m=0}^{\infty} A_{n m} t^{m}
$$

Now we come to the space of meromorphic vector fields, which are allowed to have poles only at $p$. By the Weierstrass gap theorem, for each value of $n$, except for $3 g-3$ values, there exists a meromorphic vector field with a pole of order $n$. The corresponding section is labeled by $v_{n}$, and we have

$$
v_{n}=t^{-n}+\sum_{m=-n+1}^{\infty} B_{n m} t^{m}
$$

It is now easy to show that our assumptions about the absence of gap for $\psi_{n}$ implies the existence of gap for $v_{n}$ for $n=1, \ldots, 3 g-3$ (see equation (3.15) below).

Now let us construct the conserved charges. For the $\psi_{n}$ we get (as $n$ runs from 1 to $3 g-3)$ :

$$
\frac{1}{2 \pi i} \oint_{p} \psi_{n}(t) c(t)=C_{n}=c_{-1-n}+\sum_{m=n}^{\infty} C_{n-1, m} c_{-m-2}
$$


Note that $C_{n}$ is composed of creation operators only, and so the only way it annihilates a state, is if the corresponding state is filled. Similarly for $s_{n}$ we get (as $n$ runs from 1 to

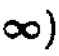

$$
\frac{1}{2 \pi i} \oint_{p} s_{n}(t) c(t)=A_{n}=c_{n-2}+\sum_{m=0}^{\infty} A_{n m} c_{-m-2}
$$

Note that $A_{1}$ is a linear sum of creation operators, and so the only way a state is annihilated is if the corresponding state is filled. The rest of the $A_{n}^{\prime}$ s are combinations of an annihilation operator with creation operators ( $A_{2}$ consists of an annihilation operator plus creation operators with respect to the $|+\rangle$ vacuum).

Similarly we can consider the conserved charges coming from the meromorphic vector fields:

$$
\frac{1}{2 \pi i} \oint_{p} v_{n}(t) b(t)=B_{n}=b_{n+1}+\sum_{m=-n+1}^{\infty} B_{n m} b_{-m+1} .
$$

Here $n$ runs from $3 g-3+1, \ldots, \infty$. $B_{n}$ 's are combinations of creation and annihilation operators.

It is easy to see that all these charges anticommute with one another. This follows because b's (and c's) anti-commute among themselves, and that if $s$ is a holomorphic quadratic differential on $\Sigma-p$ and $v$ is a holomorphic vector field on $\Sigma-p$, we have

$$
\oint_{p} v(t) s(t)=0
$$

which follows from pulling the contour off the surface, and therefore,

$$
\left[\frac{1}{2 \pi i} \oint b(t) v(t), \frac{1}{2 \pi i} \oint c(t) s(t)\right]_{+}=\frac{1}{2 \pi i} \oint v(t) s(t)=0 .
$$

Now we would like to show that these charges uniquely characterize a ray in the Hilbert space by considering the subspace of the Hilbert space annihilated by all these charges. That there is a unique ray satisfying these conditions follows from the discussion in the previous section. In this section we will give a more direct and less abstract proof of this fact.

For this purpose it is useful to give a representation of the b's and c's as operators acting on a function space. Namely, we consider the space of functions of Grassman variables $b_{-n}$ for $n \geq 0$ and $c_{-n}$ for $n>0$. We can represent the commutation relations (3.2) by

$$
b_{n} \leftrightarrow \frac{\partial}{\partial c_{-n}} \quad n>0 \quad c_{n} \leftrightarrow \frac{\partial}{\partial b_{-n}} \quad n \geq 0,
$$


and the vacuum $|+\rangle$ is represented by the function 1 . Conserved charges $A_{n}$ for $n>1$ can be written as

$$
\frac{\partial}{\partial b_{-n+2}}+\sum_{m=0}^{\infty} A_{n m} c_{-m-2}
$$

The condition of being annihilated by these uniquely fixes the $b$ dependence of the function we are looking for. Similarly, the $B_{n}$ charges determine the $c$ dependence of the functions, except for a $3 g-3+1$ dimensional subspace (because of the gaps). But the condition of being annihilated by $C_{1}, C_{3 g-3}, A_{1}$ fixes the rest by multiplying the function by them. To see that these charges correspond to the undetermined subspace of the kernel of the $B_{n}$ charges, note that $C_{n}$ and $A_{n}$ are independent, and also anticommute with one another and with $B_{n}$. So our final answer for the function is uniquely given (up to an overall normalization) by

$$
|\phi\rangle=C_{1} C_{2} \ldots C_{3 \sigma-3} A_{1} \exp \left(-\sum_{m, n=0}^{\infty} b_{-n} A_{n+2, m} c_{-m-2}\right)|+\rangle
$$

(to see that $B_{n}$ 's annihilate this state one must use (3.15)). We note that the ghost number of $|\phi\rangle$ is $3 g-3+3 / 2$. This implies that if we want to get a non-vanishing result for $\langle 0|\ldots| \phi\rangle$ we must insert an operator of ghost number $3 g-3$, i.e., we must have $3 g-3$ more $b$ 's than $c$ 's. This is again the reflection of the index theorem.

Now we consider a Riemann surface with $n$ punctures at $p_{k}$ (again for uniformity of treatment we consider $g>1$ but the sphere and torus can be handled in a similar way). In this case we should get a ray in the $n$-fold tensor product of the Hilbert space. To construct the conserved charges we should allow the quadratic differentials and the vector fields to have poles at the position of the punctures. In constructing the corresponding conserved charges, we should take the corresponding meromorphic sections restricted to the coordinate patches of each of the punctures and take a sum of contour integrals of the meromorphic sections folded in with the ghost fields around each of the punctures. For instance if $s$ denotes a quadratic differential and $b^{(k)}\left(t_{k}\right)$ denotes the ghost field acting on the $k$-th Hilbert space, the corresponding conserved charge $Q(s)$ is

$$
Q(s)=\sum_{k=1}^{n} \frac{1}{2 \pi i} \oint_{p_{k}} b^{(k)}\left(t_{k}\right) s\left(t_{k}\right)
$$

where the contours are sufficiently small to include only one puncture at a time. 
A basis for the meromorphic quadratic differentials which are allowed to have poles only at the punctures is easily chosen: we simply consider the $3 g-3$ quadratic differentials which have no poles, and append to it a basis of quadratic differentials which have a pole of a given order at a given puncture. The charges corresponding to the $3 g-3$ globally defined quadratic differentials will be denoted, as before, by $C_{1}, \ldots C_{3_{g}-3}$. The charges corresponding to the rest of the meromorphic quadratic differentials will be denoted by $A_{m}^{(k)}$, where $k$ denotes the position of the puncture where the quadratic differential has a pole, and $m$ denotes the order of the pole. Note that these are linear combination of ghost fields of different punctures. Again, as in the single puncture case, we can see that $C_{1}, \ldots, C_{3 g-3}, A_{1}^{(1)}, A_{1}^{(2)}, \ldots, A_{1}^{(n)}$ are entirely composed of creation operators $c_{-n}^{(k)}$. The rest of the $A$ 's give differential equations which fix the $b^{(k)}$ dependence of the state completely. $A$ basis for the meromorphic vector fields is more difficult to construct. One may at first think that it is sufficient to consider vector fields which have a single pole only at a given puncture. But that cannot be a complete basis, as can be seen by the fact that this would imply having $n(3 g-3)$ gaps, whereas the Weierstrass gap theorem implies that there are only $(3 g-3)$ gaps. This is because we can have meromorphic sections which have simultaneous poles at different punctures which cannot be written as a linear combination of sections which have poles only at one puncture. Again similar to the single puncture case the corresponding conserved charges give rise to differential equations for $c^{(k)}$ which determine the $c$ dependence up to a $3 g-3+n$ dimensional space, but the $C$ 's and $A_{1}$ 's uniquely fix this subspace, as in the one puncture case (where the analogue of (3.15) and (3.16) hold and are sum of contour integrals about each puncture). If we write $A_{n}^{(k)}$ as

$$
A_{n}^{(k)}=c_{n-2}^{(k)}+\sum_{m=0}^{\infty} \sum_{l=1}^{n} A_{n m}^{(k, l)} c_{-m-2}^{(l)}
$$

the corresponding ray is given by

$$
|\phi\rangle=C_{1} \ldots C_{3 p-3} A_{1}^{(1)} \ldots A_{1}^{(n)} \exp \left(-\sum_{m, n=0}^{\infty} \sum_{k, l=1}^{n} b_{-n}^{(k)} A_{n+2, m}^{(k, l)} c_{-m-2}^{(l)}\right)|+\rangle_{1} \ldots|+\rangle_{n} .
$$

It is easy to see that this state has a total ghost number of $3 g-3+\frac{3}{2} n$ (this is also true for the case of the sphere and the torus). Of course the above state does not have a definite ghost number for each Hilbert space. It is only the total ghost number, which is the sum of ghost numbers for each Hilbert space, that is conserved and is fixed in (3.18). 
In the previous section we discussed how one can glue two surfaces together and how the resulting operation is reflected at the level of Hilbert space. As discussed in section 2 the sewing is defined by the state $\left|S_{12}\right\rangle$, which is the state corresponding to the sphere with two punctures, one at 0 , and the other at $\infty$ with coordinates being $z$ and $w=1 / z$ respectively. It is easy to see using conserved charges that for the ghosts

$$
\left|S_{12}\right\rangle=\prod_{m=1}^{\infty} \exp \left(c_{-m}^{2} b_{-m}^{1}\right) \exp \left(c_{-m}^{1} b_{-m}^{2}\right)\left(b_{0}^{1}-b_{0}^{2}\right)|+\rangle_{1}|+\rangle_{2} \text {. }
$$

The sewing is now defined as in section 2. Note that $\left|S_{12}\right\rangle$ has zero total ghost number. The CTA now implies that we can obtain an arbitrary genus with punctures from the sphere with three punctures, using the sewing state defined above. In particular note that the ghost numbers add up right. For the $; \infty$; we go from $\left(g_{1}, n_{1}\right)+\left(g_{2}, n_{2}\right) \rightarrow$ $\left(g_{1}+g_{2}, n_{1}+n_{2}-2\right)$ and the ghost numbers add up $3\left(g_{1}-1\right)+(3 / 2) n_{1}+3\left(g_{2}-1\right)+(3 / 2) n_{2}=$ $3\left(g_{1}+g_{2}-1\right)+3 / 2\left(n_{1}+n_{2}-2\right)$.

This concludes our discussion of the ghost Hilbert space.

\section{Virasoro Action on Moduli Space}

It has been discovered [1] that there is a nice action of the Virasoro algebra on the moduli space of punctured surfaces, if we choose a coordinate at the puncture. In this section we will elaborate on this, in light of some concrete examples that we have discussed in the previous sections. Some of the ideas have appeared in other works[27].

Consider a Riemann surface $\Sigma$ with a puncture at $p$ and a coordinate $t$ in its neighborhood vanishing at $p$. We denote this collection of data by $P$. We assume that $t$ is a good coordinate on the unit disc $D=\{t:|t| \leq 1\}$. Consider an annulus $A$ defined by $r<|t|<1$. The Riemann surface $\Sigma$ can be viewed as being obtained from $\Sigma-(|t| \leq r)$ by attaching the disc $D$ and identifying the annulus $A$ with the corresponding points on the Riemann surface (see fig. 4.1).

Consider a meromorphic vector field $v(t) \frac{\partial}{\partial t}$ in this neighborhood which is allowed to have a pole only at $t=0$. We write

$$
v(t)=\sum a_{n-1} t^{n}
$$

We can use the vector field $v$ to obtain a new surface as follows: We deform $A \rightarrow A^{\prime}$ by (see fig. 4.1)

$$
t \rightarrow t+\epsilon v(t) \quad \text { for } \quad t \in A
$$


and we fill the inside of the annulus to obtain a new disc. We now glue this disc to $\Sigma-(|t| \leq r)$ by identifying the new annulus $A^{\prime}$ with the previous collar on the Riemann surface such that if $q^{\prime} \in A^{\prime}$ is the image of $q \in A$ under (4.1), and $q$ corresponds to a point on the surface, $\Sigma-(|t| \leq r)$, then $q^{\prime}$ is glued to $q$. In this way we get a new Riemann surface $\Sigma^{\prime}$. The question is what is the relation of the new surface with the old surface $\Sigma$ ? If $v$ is holomorphic and vanishing at $t=0$ (i.e. $a_{n-1}=0$ for $n \leq 0$ ), $\Sigma^{\prime}$ differs from $\Sigma$, only in that we have chosen a different coordinate at $p$. This is because the deformation (4.1) extends all the way inside the disc $D$. If $v$ is holomorphic, but non-vanishing at the origin (i.e., $a_{1} \neq 0$ ), The resulting surface will differ from $\Sigma$ in that the position of the puncture has moved, but the moduli of the surface does not change.

Now suppose $v$ can be extended to a holomorphic vector field on $\Sigma-p$. Then $\Sigma^{\prime}$ is identical to $\Sigma$, because the variation in the identification resulting from (4.1) can be undone by a transformation generated by $v$ on the rest of the surface. By the Weierstrass gap theorem, for $g>1$, except for a $3 g-3$ dimensional subspace, every meromorphic vector field $v$ on the disc can be written as a sum of a holomorphic one plus a vector field that extends to the rest of $\Sigma$. The $3 g-3$ dimensional subspace of vector fields which cannot be written as such a combination, correspond to infinitesimal deformations of the moduli of $\Sigma[28]$. On a generic point ( $p$ not being a Weierstrass point) we can choose a basis for this $3 g-3$ dimensional space by taking

$$
v(t)=t^{-1}, t^{-2}, \ldots, t^{-(3 q-3)} .
$$

In this way we see that we can change the moduli of the surface, change the position of the puncture, and change the coordinate at the puncture. In other words, the space of meromorphic vector fields maps onto the tangent space of punctured Riemann surfaces with coordinates at the puncture. This statement generalizes to the case of Riemann surfaces with $n$ punctures. In this case the space of $n$-tuple meromorphic vector fields at the punctures maps onto the tangent of moduli space of $n$ times punctured surfaces with coordinates at punctures. The kernel of this map consists of $n$-tuples of meromorphic vector fields which correspond to the restriction of a holomorphic vector field on the Riemann surface minus the punctures, to the coordinate neighborhood of the punctures.

Note that we can exponentiate a given deformation (4.1) by a finite amount. This simply corresponds to the action of $\exp \left(\operatorname{av}(t) \frac{\partial}{\partial t}\right)$ on $A$ which is well defined for a small. For example, if $v=t^{-k}$, we see that

$$
t \rightarrow t\left(1+\frac{(k+1) a}{t^{k+1}}\right)^{\frac{1}{t+1}}
$$


and so choosing $|a|<\frac{r^{k+1}}{k+1}$ the exponential action on $A$ is well defined. This implies that $v(t)$ not only defines a tangent vector at the point $P$, but also a vector field along a path starting at $P$. We will label the corresponding vector field by $V(v)$. It is natural to ask what is the commutator of two such vector fields. It is clear from (4.1). that if we consider deforming $A$ by $v_{1}$ and then by $v_{2}$ and then by $-v_{1}$ and then by $-v_{2}$, the effect to second order is simply $-\left[v_{1}, v_{2}\right]$. Therefore we see that the resulting Riemann surface is obtained from $\Sigma$ by deforming $A$ with the vector field $\left[v_{2}, v_{1}\right]$ which is represented by the vector $V\left(\left[v_{2}, v_{1} \mid\right)\right.$ at $P$. We have thus seen that

$$
\left[V\left(v_{1}\right), V\left(v_{2}\right)\right]=V\left(\left[v_{1}, v_{2}\right]\right)
$$

In a conformal theory, we associate a ray to a point in $P(g, 1)$, i.e., to the triple consisting of a Riemann surface and a puncture and a coordinate $t$ chosen at the puncture. We represent this ray by $|\phi\rangle$. As we discussed above every vector field corresponds to a variation of the above data (point, moduli and coordinate). Therefore it is very natural to ask what is the corresponding variation for $|\phi\rangle$ ? If we demand that this variation be given by some operator acting on $|\phi\rangle$ which we represent by $O(v)$, it is clear from (4.3) that $O(v)$ should satisfy

$$
\left[O\left(v_{1}\right), O\left(v_{2}\right)\right]=O\left(\left[v_{1}, v_{2}\right]\right)+\text { c.number }
$$

The c.number appears in (4.4) because the state $|\phi\rangle$ is only defined up to an overall normalization.

What is the right candidate for $O(v)$ ? In any conformal theory a fundamental role is played by the Virasoro algebra $L_{n}$ (and $\bar{L}_{n}$ ). We consider the energy momentum tensor, which in a given neighborhood is defined by

$$
T(t)=\sum L_{n} t^{n-2}(d t)^{2}
$$

We will now show why the Virasoro algebra is so important. For every vector field $v(t)$ in the neighborhood of $p$, we consider

$$
T(v)=\frac{1}{2 \pi i} \oint_{p} T(t) v(t)=\sum a_{-n} L_{n} .
$$

The commutation relation the $T(v)$ satisfy is

$$
\left.\left[T\left(v_{1}\right), T\left(v_{2}\right)\right]=T\left(\mid v_{1}, v_{2}\right]\right)+\frac{c}{12} C\left(v_{1}, v_{2}\right)
$$


where $c$ is the central extension of the Virasoro algebra and $C\left(v_{1}, v_{2}\right)$ is a nontrivial cocycle for the Virasoro algebra. (There is only one class [29][30].) We therefore see that $T(v)$ has the right commutation properties to be identified with $O(v)$. The fundamental property of the Virasoro algebra in any conformal theory is that the corresponding variation of $|\phi\rangle$ under the variation of the data caused by the vector field $v(t)$, is given by

$$
\delta|\phi\rangle=(T(v)+\bar{T}(\bar{v}))|\phi\rangle
$$

For the examples of conformal theories that we have discussed in this paper, we wi... show why this is true. In general, this should be taken as the defining property (together with the value of the central extension $c$ ) of the action of the Virasoro algebra.

To show that (4.7) is true for the examples that we have considered, we simply observe that the states $|\phi\rangle$ in all the examples of conformal theories we have discussed are characterized by the fact that they are annihilated by the relevant conserved charges. We will show that if $Q_{i}$ forms a basis for the conserved charges, then

$$
Q_{i} \rightarrow Q_{i}+\epsilon\left[T(v), Q_{i}\right]
$$

forms a basis for the conserved charges at the variation of the data given by $v$ for the holomorphic examples we have discussed. In the case of the scalars, since as discussed in section 2 left and right mix, the conserved charge will have anti-holomorphic degrees of freedom and the variation of the charges will require also commuting with anti-holomorphic energy momentum tensor. In what follows we will consider the holomorphic case; the other case can be handled similarly.

If we assume the variation of the conserved charge is given by (4.8), it is clear that (4.7) is true, because

$$
\left.\left(Q_{i}+\epsilon\left[T(v), Q_{i}\right]\right)(1+\epsilon T(v))|\phi\rangle\right)=\left(\epsilon Q_{i} T(v)-\epsilon Q_{i} T(v)\right)|\phi\rangle=0
$$

To show (4.8) we first show that if $s$ is a meromorphic section of a bundle on the surface $\Sigma$, which is allowed to have its poles at the puncture, then

$$
s \rightarrow s+\epsilon \mathcal{L}_{v} s
$$

is a meromorphic section of the corresponding bundle on the surface $\Sigma^{\prime}$, where $\mathcal{L}$. denotes Lie derivative with respect to the vector field $v$. We construct a section for $\Sigma^{\prime}$ by taking 
the same section $s$ over $\Sigma-(|t|<r)$. Now in order to define the section over the rest of $\Sigma^{\prime}$ we w:ll have to specify what the value of the section is on $A^{\prime}$ and the disk formed by filling it in. The value on $A^{\prime}$ is uniquely specified, because that joins the surface $\Sigma^{\prime}-(|t|<r)$ in the collar specified by $r<|t|<1$, whose value we have taken to be the previous value for the section. But since $A^{\prime}$ differs from $A$ by (4.1) it follows that the value of the section on $A^{\prime}$ is given by equation (4.10). This has a unique holomorphic extension to the whole disk which has poles only at $t=0$. This means that equation (4.10) is true on the full disk (minus the puncture).

Now suppose that $s_{i}$ forms a basis for the meromorphic sections (with poles only at p) over $\Sigma$. Then it is clear that $s_{i}-\epsilon \mathcal{L}_{v} s_{i}$ forms a basis for the meromorphic sections over $\Sigma^{\prime}$. This is clear because the variation $(4.10)$ is invertible, namely we use $-v(t)$ to go back from $\Sigma^{\prime}$ to $\Sigma$ and since $s_{i}$ forms a basis for meromorphic sections over $\Sigma$.

We get a basis for conserved charges by considering

$$
Q\left(s_{i}\right)=\oint s_{i}(t) \Phi(t)
$$

where $\Phi(t)$ denotes the field variable (of spin $j$ ). We can check in all our examples that

$$
\left[L_{n}, \Phi(t)\right]=-\left(t^{-n+1} \frac{\partial}{\partial t}+j(1-n) t^{-n}\right) \Phi(t)
$$

from which it follows that

$$
[T(v), \Phi(t)]=-\mathcal{L}_{v} \Phi(t)
$$

and so we obtain

$$
\left[T(v), Q\left(s_{i}\right)\right]=-\oint s_{i}(t) \mathcal{L}_{v} \Phi(t)=\oint\left(\mathcal{L}_{v} s_{i}\right) \Phi(t)=Q\left(\mathcal{L}_{v} s_{i}\right)
$$

We have finally seen, therefore, that if $Q\left(s_{i}\right)$ form a basis for conserved charges at $P$, then $Q\left(s_{i}\right)+\epsilon\left[T(v), Q\left(s_{i}\right)\right]$ form a basis for conserved charges at $P+\epsilon V(v)$. This concludes what was to be shown.

We discussed the case of the single puncture but it is clear that this can be generalized to the case of multiple punctures. In this case the tangent space will be spanned by $n$-tuples of meromorphic vector fields. The corresponding variation of the state with respect to an $n$-tuple is given by the action of $\sum T\left(v_{i}\right)$ on the $n$-fold tensor product of the Hilbert space, where $T\left(v_{i}\right)$ acts on the $i-t h$ Hilbert space. 
To illustrate some of these ideas, let us discuss the Virasoro action on moduli space for the particular case of spin $1 / 2$. We recall from section 2 that the state is given by

$$
|\phi\rangle=C \exp \left[\frac{-1}{(2 \pi i)^{2}} \oint \oint b(t)\left(G\left(t, t^{\prime}\right)-\frac{1}{\left(t-t^{\prime}\right)}\right) c\left(t^{\prime}\right)\right]|0\rangle
$$

where $G\left(t, t^{\prime}\right)$ is the Szego kernel and $C$ is an undetermined normalization. How do we expect $G\left(t, t^{\prime}\right)$ to change under the variation of the data represented by $v$ ? A similar case has in fact been discussed in the mathematics literature [31], whose strategy we will follow. Let $G^{\prime}\left(t, t^{\prime}\right)$ denote the corresponding Szego kernel on the Riemann surface $\Sigma^{\prime}$. Note that $\Sigma$ and $\Sigma^{\prime}$ have a common region namely the region defined by $\Sigma-(|t|<r)$. Let us denote the boundary of this common region by $S$. Then it is clear that

$$
\oint_{p \in S} G(t, p) G^{\prime}\left(p, t^{\prime}\right)=0
$$

This follows by noting that the contour could be deformed without encountering any singularities, and pulled off the surface $\Sigma-(|t|<r)$. Here $t$ and $t^{\prime}$ are two points inside the disc. On the other hand we note the following identity:

$$
\delta G\left(t, t^{\prime}\right)=G^{\prime}\left(t, t^{\prime}\right)-G\left(t, t^{\prime}\right)=\frac{1}{2 \pi i} \oint G^{\prime}\left(t, t^{\prime \prime}\right) G\left(t^{\prime \prime}, t^{\prime}\right)
$$

where the region surrounded by the contour is taken to include $t, t^{\prime}$. This equation follows by a simple application of Cauchy's integral formula, by recalling that the Green's functions have a single pole when their arguments are equal, with the residue equal to $\pm 1((4.17)$ is valid even for noninfinitesimal variations). Equation (4.17) may look contradictory with (4.16), but in fact they are not! The point is that the contour integral in (4.16) is over $S$ which corresponds to two different curves in the coordinate $t$ depending on whether we are considering $\Sigma$ or $\Sigma^{\prime}$. This is because according to (4.1) the circle $S$ in the the case of $\Sigma$ is simply $|t|=r$, but in the case of $\Sigma^{\prime}$, since we have deformed the region by (4.1), the circle which is identified with $S$ is the image of the circle $|t|=r$ under the action of the vector field $v$. This means that if we replace $G\left(t^{\prime \prime}, t^{\prime}\right)$ in $(4.17)$ by $\left(1+\mathcal{L}_{v}\right) G\left(t^{\prime \prime}\right.$, $\left.t^{\prime}\right)$ we get zero. This in turn means that

$$
\delta G\left(t, t^{\prime}\right)=-\frac{1}{2 \pi i} \oint G^{\prime}\left(t, t^{\prime \prime}\right) \mathcal{L}_{v} G\left(t^{\prime \prime}, t^{\prime}\right)
$$

To leading order we can replace $G^{\prime}\left(t, t^{\prime \prime}\right)$ by $G\left(t, t^{\prime \prime}\right)$, and so we finally obtain

$$
\delta G\left(t, t^{\prime}\right)=-\frac{1}{2 \pi i} \oint G\left(t, t^{\prime \prime}\right) \mathcal{L}_{v} G\left(t^{\prime \prime}, t^{\prime}\right)
$$


This variation of the Green's function can also be derived easily by considering the action of $T(v)$ on the state $|\phi\rangle$ and demanding

$$
(1+T(v))|\phi\rangle=(C+\delta C) \exp \left(\frac{-1}{(2 \pi i)^{2}} \oint \oint b(t)\left[G\left(t, t^{\prime}\right)+\delta G\left(t, t^{\prime}\right)-\frac{1}{\left(t-t^{\prime}\right)}\right] c\left(t^{\prime}\right)\right)|0\rangle
$$

But equation (4.20) contains more information. It also gives a variation for the normalization of the state $|\phi\rangle$ denoted by $\delta C$. Is there any way of understanding this variation? From the correspondence with the path integral we expect that $C=\langle 0 \mid \phi\rangle$ will correspond to the determinant of the $\partial \partial^{\dagger}$ operator acting on the spin bundle. It is known that the determinants do not just depend on the conformal structure of the surface, but actually on the metric chosen to represent that conformal structure. Even so, we can still ask how the determinant varies when we change the metric in some particular way and that may not depend on the metric chosen to represent a conformal class, as we will see below.

To obtain an understanding of $\delta C$ along these lines we note that there is another way we can look at the deformation which resulted in getting $\Sigma^{\prime}$. We can always view the deformation of the annulus $A$ by (4.1) as a change of coordinates inside the disk, if we allow non-analytic coordinates. This means that we can always extend $v$ from $A$ to the inside of the disk, by using non-analytic functions. The resulting extension we call $i$, and is known in the mathematics literature as a quasiconformal extension. Under such a quasiconformal deformation the only difference between $\Sigma$ and $\Sigma^{\prime}$ is in the metric chosen inside the disk. The metric on the disc changes to

$$
|d t|^{2} \rightarrow|d t+\partial \tilde{v} d t+\bar{\partial} \bar{v} d \bar{t}|^{2}
$$

This gives rise to the Beltrami differential $\mu(z, \bar{z})$ on the whole Riemann surface which is $\bar{\partial} \bar{v}$ on the disc, and is taken to vanish outside the disc.

The variation of $\log ($ det $)$ is given by the expectation value of the energy momentum tensor, namely $[32\}$

$$
\frac{1}{2 \pi i}\left\langle\int_{\Sigma} T_{\mu \nu} \delta g^{\mu \nu}\right\rangle
$$

We note that $\delta g^{\mu \nu}$ vanishes outside the disc. So we can write this in terms of integrals on the disk. Moreover since $T_{t i}$ vanishes (note that we started with flat metric on the disc), we have

$$
\delta \log (\text { det })=\frac{1}{2 \pi i}\left\langle\int_{D} T_{t t} \delta g^{t t}+\text { c.c. }\right\rangle
$$


Noting that $\delta g^{t t}=\bar{\partial} \tilde{v}$ and that $T_{t t}$ is holomorphic, we can reduce the integral in (4.23) to an integral over the boundary of the disc (on which $\bar{v}=v$ ). We obtain

$$
\delta \log (\operatorname{det})=\langle T(v)+c . c .\rangle
$$

Since the state $|\phi\rangle$ gives the correct propagator, and since the expectation value of the energy momentum tensor is obtained simply by taking derivative of the Green's function, with the leading singularity subtracted, we finally have

$$
\delta \log (\text { det })=\langle T(v)+c . c .\rangle=\frac{\langle 0|T(v)+c . c .| \phi\rangle}{\langle 0 \mid \phi\rangle}=\delta \log C .
$$

This shows that the action of the Virasoro algebra on the state correctly reproduces the effect of the variation of the determinant.

Consider now the variation of the period matrix under the deformation (4.1). Since we know what the Beltrami differential is, we can use [33] to write

$$
\delta \tau_{i j}=-\int \omega^{i}(z) \omega^{j}(z) \mu(z, \bar{z})
$$

Since $\mu(z, \bar{z})$ vanishes outside the disc, and is equal to $\bar{\partial} \tilde{v}$ inside the disc we can perform the above integral by parts to obtain a contour integral around the puncture

$$
\delta_{v} \tau_{i j}=-\oint \omega^{i}(z) \omega^{j}(z) v(z)
$$

This equation can also be checked by looking at the variation of the states (for example the tau function) under the action of the Virasoro algebra.

Now suppose that we consider a meromorphic vector field $v$ which extends holomorphically to the rest of the surface. What do we expect for

$$
T(v)|\phi\rangle=?
$$

From what we have said above it follows that this operation should be multiplication by a constant, because this vector field does not change the data, and so all it can do is to change the normalization of the state $|\phi\rangle$ (i.e., it should leave the ray invariant). If the energy momentum tensor were a well defined quadratic differential on the surface, then we would expect (4.28) to be equal to zero, by pulling off the contour. But as is well know the energy momentum tensor is not a well defined quadratic differential on the surface 32 , , so 
$T(v)$ is not a well defined object on the Riemann surface. Put differently, under a change of coordinate $z \rightarrow t(z)$ we find [2]

$$
T(z) \rightarrow T(t(z))\left(\frac{\partial t}{\partial z}\right)^{2}+\frac{c}{12}\{t, z\}
$$

where $\{t, z\}$ denotes the Schwarzian and is defined by

$$
\{t, z\}=\frac{t^{\prime \prime \prime}}{t^{\prime}}-\frac{3}{2}\left(\frac{t^{\prime \prime}}{t^{\prime}}\right)^{2}
$$

The Schwarzian is what prevents the energy momentum tensor from being a well defined quadratic differential on the surface.

One of the crucial properties of the Schwarzian derivative is that it vanishes for Mobius transformations. So for a coordinate neighborhood about a puncture, which can be joined with other patches such that the transition maps are Mobius transformations we expect to get a vanishing result for (4.28). In particular, if we use the Schottky method to obtain Riemann surfaces by gluing discs on the sphere, which are obtained by Mobius transformations, then (4.28) vanishes. ${ }^{7}$. In fact for the states that we have defined it is not difficult to see this is indeed true. All we have to check, is that for the sphere with three global coordinates at the punctures (for example, $z, z-1,1 / z$ at $(0,1, \infty)$ that the analogue of (4.28) for multiple punctured case vanishes. This means that if $v_{i}$ (with $i=1,2,3$ ) is a restriction of a holomorphic vector field on the sphere minus the punctures, to the three coordinates at the punctures, then

$$
\sum T\left(v_{i}\right)|\phi\rangle=0
$$

where $|\phi\rangle$ is a state in three fold tensor product of the Hilbert space and $T\left(v_{i}\right)$ acts on the $i$-th Hilbert space. This is easily checked in all our examples. But now, by the CTA it follows that after sewing spheres with global coordinates at the punctures together, we still obtain a Riemann surface for which (4.28) vanishes. So for coordinates corresponding to Schottky parameterization (4.28) vanishes. What other coordinates can we choose? From what we said above we expect (4.28) to vanish for coordinates which can be completed to coordinate systems on the surface with transition functions being elements of $P S L(2, C)$.

\footnotetext{
Also, if we choose coordinates coming from a uniformization of the Riemann surface, by viewing the Riemann surface as sitting in a domain in the upper half plane whose sides are identified by $S L(2, C)$ transformations, we still expect (4.28) to vanish [11].
} 
But what is the dimensionality of this space? Suppose we start from a coordinate choice where (4.28) vanishes. We can consider a small change of coordinates in a given patch represented by $\epsilon(z)$. To preserve (4.28) we must make sure that the central term does not contribute to the contour integral. This means that we must have

$$
\oint \epsilon^{\prime \prime \prime}(z) v(z)=0
$$

for all $v(z)$ which extend holomorphically to the rest of the surface. This means that $\epsilon^{\prime \prime \prime}(z) \in H^{0}\left(\Sigma, K^{2}\right)$, i.e., $\epsilon^{\prime \prime \prime}(z)$ should be the expansion of a globally defined quadratic differential in the coordinate neighborhood. The dimension of the space of solutions to this differential equation is $3 g-3+3=3 g$, where $3 g-3$ comes from the choice of the quadratic differential and +3 comes from the fact that the differential equation has order 3 (and corresponds to $P S L(2, C)$; a suitable notion of equivalence removes these three dimensions.) We can also write the exponentiated version of this differential equation. Using (4.29) we find that if $t(z)$ represents the coordinate transformation then

$$
t^{\prime \prime \prime}(z) t^{\prime}(z)-\frac{3}{2}\left(t^{\prime \prime}\right)^{2} \in H^{0}\left(\Sigma, K^{2}\right)
$$

Coordinate systems with $P S L(2, C)$ transition functions on a given Riemann surface are (modulo equivalence) known as projective structures. From the above considerations we expect that the set of projective structures is $3 g-3$ dimensional ${ }^{8}$. It would also be interesting to investigate the solution space to (4.31). It has indeed been considered ${ }^{2}$ in the mathematics literature in connection with Teichmuller space [34]. It seems likely that the space of good coordinates at a point is isomorphic to the Teichmuller space itself. If this is so it seems to suggest a nice duality between the moduli choices, and the choices for good coordinates. It would be worthwhile to understand this connection more clearly.

Let us briefly summarize the intuition for dealing with the variation of the state on moduli space, for the case of a single puncture which is not a Weierstrass point. Then we have

$$
\begin{gathered}
L_{-n}|\phi\rangle \text { changes the coordinates for } n \geq 0 \\
L_{1}|\phi\rangle \text { moves the puncture }
\end{gathered}
$$

8 It can be shown that the set of projective structures is naturally embedded in $H^{1}(\Sigma, P S L(2, C)) \cong \operatorname{Hom}\left(\pi_{1}(\Sigma), P S L(2, C)\right) / P S L(2, C)$. For an explanation of this and related matters see section 9 of $(17)$.

- We would like to thank D. Kazhdan for pointing out this connection. 


$$
L_{k}|\phi\rangle \text { changes the moduli for } k=2, \ldots, 3 g-3+1 \text {. }
$$

The rest of the $L_{n}$ 's can be written as linear combinations of the above (apart from a constant), as follows from the fact that (4.28) action is multiplication by a constant for vector fields which extend to the rest of the surface (if the puncture is a Weierstrass point the basis for moving on moduli space is different from (4.34), but that could also be easily written, depending on the space of meromorphic vector fields at the puncture, which extend to the rest of the Riemann surface).

Let us also note that from what we said about exponentiating the action (4.1), it follows that we can exponentiate the Virasoro algebra for a finite amount, depending on the radius of the convergence of the coordinate. So the state

$$
\exp \left(a L_{n}\right)|\phi\rangle
$$

is well defined for small enough $|a|$. This does not give rise to a group structure, in general, because we cannot exponentiate it beyond a certain amount where $|a|$ gets too large, and the exponentiated version of (4.1) ceases to have a well defined geometrical interpretation (more precisely this shows that a representation of the Virasoro algebra cannot in genera! be exponentiated, but there is a stronger statement [16] which implies that there is no complex Lie group with the Virasoro algebra as its Lie algebra). But the fact that we can exponentiate by a finite amount, and not just infinitesimally, implies that we can move the state $|\phi\rangle$ over the moduli space of punctured surfaces with coordinates chosen at the punctures.

We close this section with two simple illustrations of the above ideas. First, let us rederive a well-known formula for the fermion determinant. From the operator form of the fermion state (2.18) we can use the Virasoro algebra to compute the variation

$$
\delta_{n} \log C=\sum_{m=1}^{n}\left(m-\frac{n+1}{2}\right) B_{n-m+1 . m}
$$

corresponding to a tangent vector $t^{-n+1} \frac{d}{d t}$. For the case of $g=1$, the modulus comes from $n=2$, and we can choose coordinates with Mobius transformations on the overlaps so we can compute

this gives

$$
\delta_{2} \log C=B_{1,2}
$$

$$
\frac{\partial}{\partial \tau} \log C \propto \frac{\partial}{\partial \tau} \log \frac{\vartheta\left[\begin{array}{l}
0 \\
\phi
\end{array}\right]}{\eta}
$$


which is a well known result for the fermionic determinant.

As a second application, we write out explicitly the transport equations for the case of free bosons. It is convenient to let indices $n, m$ on $Q$ etc. run over the integers, so we define:

$$
\begin{array}{rllll}
Q_{n m}=0 & \text { if } & n \leq 0 & \text { or } & m \leq 0 \\
A_{n}^{i}=0 & \text { if } & n \leq 0 & &
\end{array}
$$

Define

$$
<x\left|L_{n}\right| \phi>\equiv \delta_{n}(C \tau(x))
$$

with

$$
L_{n}=\frac{1}{2} \sum_{r}: j_{n-r} j_{r}:
$$

Then the transport equations are

$$
\begin{aligned}
\delta_{n} \log C & =-Q_{r, n-r} \\
\delta_{n} A_{p}^{i} & =-2 Q_{r p} A_{n-r}^{i}+p A_{n+p}^{i} \\
\delta_{n} \tau_{i j} & =-2 \pi i A_{n-r}^{i} A_{r}^{j} \\
\delta_{n} Q_{p q} & =-2 Q_{r p} Q_{n-r, q}+p Q_{n+p, q}+q Q_{n+q, p}-\frac{1}{2} p q \delta_{n+p+q}
\end{aligned}
$$

where repeated indices are summed.

\section{Polyakov string}

In this section we will present an operator formulation of Polyakov strings which makes sense on an arbitrary genus surface. We will combine the results of the previous sections to show that for an arbitrary process involving physical particles, we get a well defined measure on the moduli space of punctured surfaces. The Polyakov measure has been considered in the operator formulation in the literature previously (for recent literature see $[35][36]$.) Our approach could be viewed as a generalization of these approaches in that modular invariance is manifeat in our formulation.

We consider 26 bosons, which correspond to strings propagating in a 26 dimensional background flat space. The Hilbert space of a single boson was described in section 2 . We simply take 26 copies of $i t$. The corresponding states coming from punctured Riemann surfaces, will also be 26 -fold tensor products of the ones described in section 2 . Moreover we tensor in the ghost system described in section 3. For the total system, we see that 
the total Virasoro algebra, which is the sum of the Virasoro algebras of the 26 bosons plus the ghosts, has no central extensions $(26 \cdot 1-26=0)$. This in particular implies that the energy momentum tensor is a well defined quadratic differential whose transformations under the changes of coordinates does not pick up the Schwarzian term in (4.29).

We will be interested in the moduli space for $n$ times punctured surfaces of genus $g$, with a choice of coordinates at the punctures. This space will be denoted by $P(g, n)$. Consider a point $P \in P(g, n)$. The corresponding state, which is in the $n$-fold tensor product of the ghost states tensored with an element in the $n$-fold tensor product of the bosonic state, will be labeled by $|\phi\rangle_{P}$. Of course the absolute normalization of $|\phi\rangle_{P}$ is not well defined, but we need to show that as $P$ varies in $P(g, n)$, we will be able to fix the relative normalizations of the state unambiguously. That this in fact can be done has been shown in [13]. From what we have described in the previous sections, it is possible to show that all the conditions required in [13] for a well defined conformal theory are satisfied. Let us review this argument. The basic strategy is to use the Virasoro algebra to find how $|\phi\rangle_{P}$ varies, including its normalization, as we move the point $P$, as discussed in the last section. There are several things we must show. We first show that if $v$ represents the restriction of a holomorphic vector field on the surface with punctures removed, then

$$
T(v)|\phi\rangle=0
$$

in an abbreviated notation, where $T(v)$ denotes the sum of the total Virasoro algebras at each puncture folded in with the corresponding vector field at each puncture. This must be shown, otherwise such $v$ 's which do not move the point $P$, will change the normalization of $|\phi\rangle$ at $P$, which means that we cannot unambiguously assign a normalization at $P$. That there exists a coordinate where (5.1) is true follows from what we said in the last section (see equation (4.30) and the comments following it). But since in this case the energy momentum tensor transforms as a true quadratic differential as we change the coordinates, the equation (5.1) remains true for an arbitrary choice of coordinates.

Starting from a point $P$, we define the normalization of the state at other points by following a path in $P(g, n)$. The second thing we must show is that going along different paths does not lead to different normalizations for the state. This is true for infinitesimal loops, since the Virasoro algebra has zero central extension (i.e, if we follow an infinitesimal loop described by the vector fields $v_{1}, v_{2},-v_{1},-v_{2},\left[v_{1}, v_{2}\right]$, then we come back to the same state with the same normalization). Moreover for contractible loops since the 
loop can be decomposed to smaller loops the above argument continues to show that we get a well defined normalization. The only conceivable difficuity occurs if we could go from $P$ to another point $Q$, via two different paths that cannot be deformed to one another. Fortunately, there are no such paths for genus bigger than 2 (see [37] and [13]) since $H_{1}(P(g, n))=0$. This shows that the relative normalizations of states are well defined for $g>2$. For the states at lower $g$, we can use the sewing operations to determine the relative normalizations unambiguously. Also the relative normalizations between different genera and punctures can be fixed by the sewing operations. These require some arguments, which we leave to the reader.

We will now describe how we obtain the Polyakov measure (for simplicity we consider the case $g>1$, the operator formulation for the other cases can be done in the standard way). Consider a basis for holomorphic tangent vectors $\tilde{V}_{1}, \ldots, \bar{V}_{3 \rho-3}$ at a point $\tilde{P} \in M_{g}=$ $P(g, 0)$. We have to show how we can obtain a number corresponding to the volume element determined by these vectors, which we denote by

$$
\mu(\tilde{P})\left(\tilde{V}_{1}, \ldots, \tilde{V}_{3 g-3}, \overline{\tilde{V}}_{1}, \ldots, \bar{V}_{3 g-3}\right) .
$$

There is a projection map

$$
\pi: \quad P(g, 1) \longrightarrow M_{0}
$$

which is obtained by ignoring the puncture and the coordinate chosen at the puncture. Of course, a given point $\tilde{P} \in M_{0}$ does not determine a unique point on $P(g, 1)$, but an infinite number of them, which differ by the choice of the position of the puncture, as well as the coordinate chosen at the puncture. Pick a point $P \in P(g, 1)$ which corresponds to $\bar{P}$ (i.e., $\pi(P)=\bar{P})$. We can choose a number of vectors $V_{1}, \ldots, V_{3 g-3}$ at $P$, which get mapped to the $\bar{V}_{i}$ vectors under the projection map $\pi$. Again, the choices for $V_{1}, \ldots, V_{3 g-3}$ are not unique. To each one, we can add a vector, which corresponds to infinitesimal changes of coordinates and the position of the puncture.

We recall from the last section, that for each tangent vector $V$ at $P$, there exists a vector field at the punctured Riemann surface which is defined in a neighborhood of the puncture. Let $v_{i}$ denote the corresponding tangent vector fields to the $V_{i}$. Again this is not one to one, and we have the freedom of shifting the $v_{i}$ by vector fields which extends holomorphically to the rest of the surface.

We now define the measure

$$
\mu(\dot{P})\left(\bar{V}_{1}, \ldots, \tilde{V}_{3 v-3}, \bar{V}_{1}, \ldots, \bar{V}_{3 g-3}\right)=\left\langle 0\left|b\left(v_{1}\right) \ldots b\left(v_{3 y-3}\right) \bar{b}\left(\bar{v}_{1}\right) \ldots \bar{b}\left(\bar{v}_{3 y-3}\right)\right| \phi\right\rangle_{P}
$$


where $b(v)$ (and similarly for its conjugate) is defined by

$$
b(v)=\oint b v
$$

Note that as discussed in section 3 , we need to put $(3 g-3) b$ 's (and similarly for the complex conjugate) to get a non-zero matrix element for $(5.4)(|\phi\rangle$ has ghost number $3 g-3+3 / 2$ and $\langle 0|$ has ghost number $-3 / 2)$. We will have to show that (5.4) is independent of the choices we have made in its construction. First we show that if we add to $v_{i}$ a vector $v$ which is holomorphic on the rest of the surface, then

$$
\begin{gathered}
b\left(v_{i}+v\right) \ldots|\phi\rangle= \pm \ldots b\left(v_{i}+v\right)|\phi\rangle \\
= \pm \ldots\left(b\left(v_{i}\right)+b(v)\right)|\phi\rangle= \pm \ldots b\left(v_{i}\right)|\phi\rangle \\
=b\left(v_{i}\right) \ldots|\phi\rangle
\end{gathered}
$$

where we used the fact that $b(v)$ is a conserved charge and thus annihilate $|\phi\rangle$. We next show that changing $V_{i}$ by the addition of vectors which correspond to infinitesimal changes of the coordinate and the position of the puncture, does not affect the measure. The variation of $V_{i}$ results in a variation of $v_{i}$, by vector fields which are holomorphic near the puncture. That there is no variation for the measure now follows by an argument similar to (5.5) where this time the corresponding conserved charge will be taken to the left where they annihilate the vacuum.

Finally we have to show that the choice of the point $P$ which mapped to the point $\tilde{P}$ does not affect the measure. For that we note that for a variation of the positions of the puncture or the coordinate at the puncture represented by $v$, we have normalized $|\phi\rangle$ such that it goes to

$$
|\phi\rangle_{P} \rightarrow|\phi\rangle_{P}+T(v)|\phi\rangle_{P}
$$

Moreover, in the new coordinate and puncture the corresponding vectors $v_{i}$ move to

$$
v_{i} \rightarrow v_{i}+\left[v, v_{i}\right]
$$

and finally, we note that

$$
\left[T(v), b\left(v_{i}\right)\right\}=b\left(\left\{v, v_{i}\right]\right)
$$

Equations (5.6)(5.7) and (5.8) together imply that the position of the puncture and the coordinates chosen do not affect the measure (5.4)(by moving $T(v)$ to the left, picking 
up commutators along the way and noting that $T(v)$ annihilates the vacuum on the left). This finally establishes that (5.4) is a well defined measure on $M_{g}$. Next we show that it is indeed the Polyakov measure.

We show the equivalence with the Polyakov measure in two ways: Our first derivation presupposes the correspondence between the path-integral and the operator formulation. Our second demonstration is independent of this correspondence, and simply shows that the conditions which characterize the Polyakov measure, as established in [38], are satisfied.

We first transform the usual Polyakov path-integral to a form convenient for us. In the usual formulation of the Polyakov measure, we have to choose $3 g-3$ Beltrami differentials $\mu_{i}$ which span the holomorphic tangent space to moduli space. Any such Beltrami differential represents the same tangent vector even after being shifted by $\mu_{i} \rightarrow \mu_{i}+\bar{\partial} v$ for a globally defined vector field (not necessarily holomorphic). We consider a point $p$ on the surface $\Sigma$ with a coordinate $t$ around that point with the discs $D_{r}:|t| \leq r$. We can always find a vector field $u_{i}$ on $\Sigma-D_{r}$ such that in that region $\bar{\partial} u_{i}=\mu_{i}$. Moreover, inside a disc $D_{r^{\prime}}$ with $r^{\prime}>r$ we can find vector fields $w_{i}$ such that $\bar{\partial} w_{i}=\mu_{i}$. In this way we obtain a holomorphic vector field $v_{i}$ on the annulus region $D_{r} \cap D_{r^{\prime}}$, defined by $v_{i}=w_{i}-u_{i}$. Of course, $v_{i}$ does not extend holomorphically inside the disc and will have singularities at $t=0$. These $v_{i}$ 's vary the moduli of the Riemann surface, in precisely the way we described in the last section.

In the Polyakov path-integral the measure is [39]

$$
Z=\int D x|D b D c|^{2}\left|\prod_{i=1}^{3 g-3} \int b(z) \mu_{i}(z, \bar{z})\right|^{2} \exp \left(\int b \bar{\partial} c+c . c .+\partial x \bar{\partial} x\right)
$$

The $b$ insertions that have been made can be simplified. We represent the Beltrami differentials in the region $\Sigma-D_{1}$ by $\bar{\partial} u_{i}$ and on the region $D_{1}$ by $\bar{\partial} w_{i}$, and then we do the integrals in (5.9) by parts to obtain

$$
\int b(z) \mu_{i}(z, \bar{z})=\oint b(z) v_{i}(z)
$$

So we have recovered the contour integrals that we have encountered in the operator formulation. The state $|\phi\rangle$ can be identified with the state obtained by path-integral on the circle $|t|=1$. Furthermore, if we assume the connection between the energy momentum tensor in the path-integral, with the operator form of it, then it is clear that under the (4.1) the state $|\phi\rangle$ varies according to (4.7). Also we have shown (5.10) that the insertion of 
the $b$ integrals on the surface can be reduced to the contour integrals that we have already encountered. This justifies the identification of (5.4) with (5.9).

We give another derivation which does not use any assumptions about the identification of the path-integration with the operator formalism. We will show the the measure (5.4) is of the form

$$
\tilde{\mu}=(\rho \wedge \bar{\rho})(\operatorname{det} \operatorname{Im} \tau)^{-13}
$$

where $\rho$ is a holomorphic $3 g-3$ form on the moduli space. This together with the right pole structure in the degenerating limit of the Riemann surface, which we will establish below, shows that it is indeed the Polyakov measure [38]. To show that (5.4) is of the form (5.11), we will have to show that, once we fix some holomorphic basis in a neighborhood of a point $\tilde{V}_{i}$, that

$$
\begin{gathered}
\partial \bar{\partial}\left(\log \left(\mu\left(\ldots, \tilde{V}_{i}, \ldots\right)\right)=\partial \bar{\partial} \log \left(\tilde{\mu}\left(\ldots, \tilde{V}_{i}, \ldots\right)\right.\right. \\
=-13 \partial \bar{\partial} \operatorname{tr}(\log (\operatorname{Im} \tau))
\end{gathered}
$$

It is clear from the definition of the measure (5.4) that

$$
\begin{gathered}
\partial \bar{\partial}\left(\log \mu\left(\ldots, \bar{V}_{i}, \ldots\right)=\right. \\
=\frac{\langle 0|\ldots| \phi\rangle\langle 0|\ldots T() \bar{T}()| \phi\rangle-\langle 0|\ldots T(\quad)| \phi\rangle\langle 0|\ldots \bar{T}(\quad)| \phi\rangle}{(\langle 0|\ldots| \phi\rangle)^{2}}
\end{gathered}
$$

where the dots on the right hand side refer to $b\left(v_{i}\right)$ insertions. The above equation defines a $(1,1)$ form. To get a number for the above equation the energy momentum tensors have to be folded in with vectors. It is clear that (5.13) would have vanished if the left-moving and right-moving Hilbert spaces did not mix. The only reason that this does not vanish is that in the bosonic Hilbert space, as discussed in section 2, left and right mix in $g$ directions. The above equation, together with the explicit form of the bosonic contribution to $|\phi\rangle$, and the variation of the period matrix $r$ under the action of Virasoro discussed in the last section (4.27), can now be used to show that equation (5.12) is true. We leave the verification of this to the reader.

The behavior of the measure (5.4) in the degenerating limit is also not difficult to see. However it is convenient first to describe equivalent ways of writing the measure (5.4) in terms of surfaces with more punctures. Consider a point $Q \in P(g, n)$. The corresponding state, has ghost number $3 g-3+\frac{3}{2} n$. To get something non-zero for

$$
\langle 0|\ldots| \phi\rangle_{Q}
$$


where $\langle 0|=\Pi_{k} k\left\langle\left. 0\right|_{k}\right.$ and $k$ labels the punctures, we have to insert operators which have total ghost number $3 g-3$. Now we would like to see how we can obtain the measure by inserting operators with the right total ghost number, but with ghost number receiving contribution from ghost fields at different punctures.

We first recall that we can vary the moduli of the surface by choosing $3 g-3$ meromorphiz vector fields near a puncture as described before. Equivalently we can chonse a total of $3 g-3$ meromorphic vector fields $w_{i}$, each defined in the neighborhood of some puncture $p_{k}$, and use them to change the moduli of the surface by the local deformations (4.1) defined near the punctures. We claim that the measure (5.4) is the same as

$$
\mu=\left\langle 0\left|\ldots b^{k}\left(w_{i}\right) \ldots\right| \phi\right\rangle_{Q}
$$

where $b^{k}$ denotes the ghost field acting on the Hilbert space of the $k$-th puncture. Fix a puncture $p$. For each of the $3 g-3$ vector fields $w_{i}$ near $p_{k}$ we look for a vector field $v_{i}$ in the neighborhood of $p$ that describes the same moduli deformation. If there exists a vector field which is meromorphic on the surface, with poles allowed only at $p$ and $p_{k}$, such that near $p$ it reduces to $v_{i}$ and near $p_{k}$ it reduces to $-w_{i}$, then $v_{i}$ and $w_{i}$ describe the same deformation. According to the discussion in section three regarding the conserved charges, it follows that

$$
\left\langle 0\left|\ldots b^{k}\left(w_{i}\right)\right| \phi\right\rangle_{Q}=\left\langle 0\left|\ldots b\left(v_{i}\right)\right| \phi\right\rangle_{Q}
$$

where $b$ is the ghost field acting on the Hilbert space corresponding to the puncture $p$ (the contribution of the conserved charges involving other punctures vanishes by taking them to the left where they annihilate the vacuum). In this way we can rewrite the measure (5.14) in terms of ghost fields of the puncture at $p$, i.e.,

$$
\left(\left\langle 0\left|\ldots b\left(v_{i}\right) \ldots \prod_{p_{i} \neq p} k\langle 0|\right) \mid \phi\right\rangle_{Q}\right.
$$

This can be simplified by noting that

$$
\prod_{p_{k} \neq p} k\langle 0 \mid \phi\rangle_{Q}=|\phi\rangle_{P}
$$

where $P \in P(g, 1)$ is the point obtained from $Q$ by ignoring all the punctures except for $p$. This equation follows from CTA described in section 2 (together with the normalization discussed in this section). Finally this completes the demonstration of the equivalence of (5.14) with (5.4). 
We have now seen how we can localize the variation of moduli on different punctures. In particular we can obtain the Polyakov measure on an arbitrary genus by first obtaining the state corresponding to the sphere with three punctures (with global coordinates at the punctures), and then sew the vertices with propagators inserted

$$
\ldots \frac{d q d \bar{q}}{q \bar{q}}\left\langle S_{i j}\left|b_{0} \bar{b}_{0} q^{L_{0}} \bar{q}^{\bar{L}_{0}}\right| \phi\right\rangle
$$

through which the $3 g-3$ parameters of the moduli space can be locally described by $3 g-3$ propagator parameters $q_{i}$. We can justify the form of the measure written in (5.16) by our previous arguments (noting that (4.7) implies that the insertions of $q^{L_{0}} \bar{q}^{\bar{L}_{0}}$ in (5.16) dilates the coordinates before being sewn together). This justifies, in particular, the prescription which has appeared in the literature (see e.g. (36]), for obtaining the Polyakov measure. It also helps us establish that (5.4) is indeed the Polyakov measure obtained from path integral, because the degeneration limit of Riemann surfaces can be described by putting punctures at appropriate places on the Riemann surface of lower genus, and sewing them together. The limit of $q \rightarrow 0$ in equation (5.16) will give the degeneration limit, and so the lightest state contributes the leading singularity to the measure, and that has $L_{0}=\bar{L}_{0}=-1$ and so equation (5.16) gives the right singularity structure $\left|d q / q^{2}\right|^{2}$. This finally completes the identification of (5.4) with the Polyakov measure (5.9). Similar arguments deriving the asymptotic behavior of Polyakov's measure have appeared in [4][21].

Now we discuss the scattering amplitudes. Which states in the Hilbert space could correspond to physical particles? Instead of stating what physical states are, we shall 'derive' what could be labeled physical in terms of being able to compute a well defined scattering amplitude. For a scattering involving $n$ particles, we would be interested in surfaces with $n$ punctures. The result of the scattering amplitude in the $g$-th order of string perturbation theory should be expressible as a measure on $\mathcal{M}(g, n)$, the moduli space of $n$ times punctured Riemann surfaces (without any choices of coordinates at the punctures). However, we are given states $|\phi\rangle$ for each point $P \in P(g, n)$ which includes the coordinates chosen at the puncture. Take $n$ states $\left|\chi_{i}\right\rangle$ in the Hilbert space. We could try to compute a scattering amplitude of these states by defining a measure similar to (5.4) by considering:

$$
\left\langle x_{1}\right| \ldots\left\langle x_{n} b() \ldots . \bar{b}() \mid \phi\right\rangle_{P}
$$


to get a measure on $\mathcal{M}(g, n)$ we need a $(3 g-3+n, 3 g-3+n)$ form. This means that there must be $(3 g-3+n) b$ 's in the above equation. In other words we need one extra $b$ per particle compared to Polyakov's measure. To get something non-vanishing, we must preserve the ghost number, which implies, together with the fact that $|\phi\rangle$ has ghost number $3 g-3+\frac{3}{2} n$ that (if they are taken to have equal ghost number) that each $\left\langle\chi_{i}\right|$ has ghost number $-1 / 2$. Next we have to make sure that (5.17) is independent of choices made, and thus gives rise to a well defined measure on $\mathcal{M}(g, n)$. The arguments leading to the well definedness of the measure discussed for (5.4) goes through as before, except that the coordinate independence at the punctures requires that

$$
\begin{gathered}
L_{n}\left|\chi_{i}\right\rangle=\bar{L}_{n}\left|\chi_{i}\right\rangle=0 \\
b_{n}\left|\chi_{i}\right\rangle=\bar{b}_{n}\left|\chi_{i}\right\rangle=0 \quad \text { for } n \geq 0
\end{gathered}
$$

(we leave checking that the above conditions are the only ones required for the well definedness of the measure to the reader). In the case of (5.4) we also needed that the position of the puncture should be immaterial, which meant that we required that the above equation should also hold for $L_{-1}$, giving us as solutions the vacua. Here since the measure is obviously going to have to depend on the choice of position of the puncture we do not require $(5.18)$ for $L_{-1}$.

The condition that $\left|\chi_{i}\right\rangle$ has ghost number $-1 / 2$ together with $(5.18)$ are solved by (dropping the index)

$$
|\chi\rangle=\sum|\psi\rangle_{\text {matter }} \otimes|-\rangle_{\text {ohoot }}
$$

where

$$
\begin{gathered}
L_{n}|\psi\rangle=\bar{L}_{n}|\psi\rangle=0 \quad \text { for } n>0 \\
L_{0}|\psi\rangle=\bar{L}_{0}|\psi\rangle=|\psi\rangle
\end{gathered}
$$

These condition imply that $|\psi\rangle$ is a primary state of the matter in the bosonic Hilbert space. In the Polyakor case, when we take the signature of spacetime to be $(25+, 1-)$, the Hilbert space inherits an indefinite metric structure from space-time. For questions of unitarity it is important to identify the physical particles as states which have positive norm. As is well known [40]|41]the definition of physical state (5.20)(5.21) implies that they have positive semi-definite norm. The physical states which have zero norm are called spurious physical states, and do not correspond to physical particles. We will have to show 
that they decouple from scattering amplitudes. This will be shown below after we discuss BRST cohomology.

Let us get a better feeling for (5.17). We recall some of the properties established in the last section for the ghost states. In (5.17) $3 g-3$ of the $b$ 's are inserted to absorb the ghost charges $C_{1}, \ldots, C_{3 g-3}$ coming from holomorphic quadratic differentials, and $n$ of them absorb the ghost charge for $A_{1}^{(i)}$, which are responsible for moving the puncture. Roughly speaking $C_{i}$ looks like $c_{-i-1}+\ldots$ and $A_{1}^{(i)}=c_{-1}^{i}+\ldots$ and the $b$ 's inserted in (5.17) are folded into vector fields which give $b_{1}^{i} b_{2} \ldots b_{30-3+1}+\ldots$ where $b_{1}$ absorbs the $A_{1}$ ghost charge and $b_{2} \ldots b_{3 g-3}$ absorb the ghost charges of $C_{1} \ldots C_{3 g-3}$ (as discussed before, they could be sitting at one puncture or be some combinations at different punctures).

Why is (5.17) the correct measure for the scattering amplitudes in Polyakov strings? This can be justified from path integral approach. From [5] we know that we can convert the scattering amplitudes, to integrations over moduli space of punctured surfaces by writing the vertex operators as

$$
\frac{1}{2 \pi i} \oint_{z} b\left(z^{\prime}\right) v\left(z^{\prime}\right) c V(z)=v(z) V(z)
$$

This together with arguments similar to the one given for showing that (5.4) is the same as the path-integral measure, justifies (5.17) as the measure for scattering amplitude. We could also try to prove more abstractly that (5.17) is the correct scattering amplitude, by generalizing the Belavin-Knizhnik theorem for scattering amplitude measures on moduli space of $\mathcal{M}(g, n)$. However, we will not pursue this here.

The main point to emphasize is that the operator formulation for the Polyakov string as we have developed it here, even though motivated from the path-integral approach, is by itself a self-contained system which is phrased with no particular reference to any path-integral and the operator formulation discussed here can be taken to be the definition of Polyakov strings. The advantage of this way of formulating the Polyakov string is that many properties are clearer in this formulation. In particular, we have formulated what an arbitrary physical amplitude is, without having to write down vertex operators for each of the states. Moreover, we are dealing with all possible scattering amplitudes at once, and factorization properties are clearer in this formulation.

Now we come to a discussion of BRST cohomology. First we recall [41] what a BRST operator is. The BRST charge is defined to be

$$
Q=c_{n}\left(L_{-n}^{\text {matter }}+\frac{1}{2} L_{-n}^{\text {ghoot }}\right)
$$


with an appropriate normal ordering of the second term. It has ghost number 1 , and has two important properties which can be taken to be its defining properties:

$$
\begin{gathered}
{[Q, b(v)]=T(v)} \\
Q^{2}=0 .
\end{gathered}
$$

It is not too difficult to show that the states that we have obtained from the path-integral are annihilated by the BRST operator. This follows from the considering the case of the sphere with up to three punctures, and using the CTA, to show it is true for an arbitrary surface. On the sphere this can be shown by writing $Q$ as the charge corresponding to a conserved current (for the case of Polyakov see e.g. (36]) Using the $Q$ operator it is possible to define a cohomology called the BRST cohomology, by viewing $Q$ as the analog of a $d$ operator in an ordinary cohomology, here acting on the Hilbert space of matter combined with ghost (we shall see below that it is indeed the $\partial$ operator acting on forms on moduli space). It turns out [41] that non-trivial elements in this cohomology are in 1-1 correspondence with physical particles. It is clear that physical states which are defined by $(5.20)(5.21)$ are closed forms of this cohomology (i.e., are annihilated by $Q$ ). What is not so clear, but is true, is that if the signature of spacetime is taken to be $(25+, 1-)$ any closed form which is not exact (i.e., which cannot be written as $Q$ acting on some state) with ghost number $-1 / 2$ can be represented by a subset of physical states defined by $(5.20)(5.21)$ which are not spurious. The zero norm physical states (which do not correspond to physical particles, but generate the gauge symmetries of the theory) can be written as exact forms (i.e. as $Q|\lambda\rangle)$, and therefore correspond to trivial elements of the cohomology. Using this fact we will now show that spurious physical states decouple from the scattering amplitudes by demonstrating that the resulting scattering amplitudes could be written as $\partial \omega$ where $\omega$ is a well defined form (of degree $(3 g-3+n-1,3 g-3+n)$ ) on $M(g, n)$. Note that every form is locally $\partial$ of some lower form. By "total derivative" we mean it is the derivative of a globally well defined form on $M(g, n)$. Once this is known then the scattering amplitudes which involve spurious physical states can by Stokes theorem be related to boundary integrals of moduli space, which can then possibly be shown to vanish. Of course in the bosonic string theories the measure is divergent precisely near the boundaries, so we cannot really talk about scattering amplitudes beyond the tree level. But we will go through the exercise of showing why the scattering amplitudes of spurious physical states are total derivatives for the corresponding case in the superstring, where we 
do expect the boundary contributions to integrate to zero. Moreover we shall see the sense in which the $Q$ operator is an exterior derivative on differential forms on moduli space. In showing this we ignore (for simplicity) the antiholomorphic parts, since the problem clearly factorizes between holomorphic and anti-holomorphic Hilbert spaces. Also, for simplicity we consider the case of one puncture.

We now discuss the decoupling of spurious states in the operator formalism. Suprose $|\chi\rangle=Q|\lambda\rangle$ is a spurious physical state. From the explicit formulas for $|\lambda\rangle\langle 42\}$ we see that we can choose a $|\lambda\rangle$ such that for $n \geq 0$ we have $L_{n}|\lambda\rangle=b_{n}|\lambda\rangle=0$.

The scattering amplitude (5.17) involving the spurious state, can be written as

$$
\mu\left(V_{1}, \ldots, V_{3 g-3+1}\right)=\left\langle\lambda\left|Q b\left(v_{1}\right) \ldots b\left(v_{3 g-3+1}\right)\right| \phi\right\rangle .
$$

By taking $Q$ all the way to the right and noting that it annihilates $|\phi\rangle$, and that along the way we pick up commutators according to (5.23), we see that

$$
\begin{gathered}
\mu=\left\langle\lambda\left|T\left(v_{1}\right) b\left(v_{2}\right) \ldots b\left(v_{3 g-3+1}\right)\right| \phi\right\rangle \\
-\left\langle\lambda\left|b\left(v_{1}\right) T\left(v_{2}\right) \ldots b\left(v_{3 g-3+1}\right)\right| \phi\right\rangle \\
+\ldots \pm\left\langle\lambda\left|b\left(v_{1}\right) b\left(v_{2}\right) \ldots T\left(v_{3 g-3+1}\right)\right| \phi\right\rangle .
\end{gathered}
$$

In each of the terms in this expression we will move $T\left(v_{i}\right)$ all the way to the right and in this way pick up commutators in accordance with (5.8). So for example the first term becomes

$$
\begin{gathered}
\left\langle\lambda\left|b\left(\left[v_{1}, v_{2}\right]\right) b\left(v_{3}\right) \ldots b\left(v_{3 g-3+1}\right)\right| \phi\right\rangle \\
+\left\langle\lambda\left|b\left(v_{2}\right) b\left(\left[v_{1}, v_{3}\right]\right) \ldots b\left(v_{3 g-3+1}\right)\right| \phi\right\rangle \\
+\ldots+\left\langle\lambda \lambda\left|b\left(v_{2}\right) b\left(v_{3}\right) \ldots\right| b\left(v_{1}\right), b\left(v_{3 g-3+1}\right)|| \phi\right\rangle \\
+\left\langle\lambda\left|b\left(v_{2}\right) b\left(v_{3}\right) \ldots b\left(v_{3 g-3+1}\right) T\left(v_{1}\right)\right| \phi\right\rangle .
\end{gathered}
$$

Since $\langle\lambda|$ is a primary state of the Virasoro algebra, it follows that we can define a we!l defined $3 g-3$ form $\omega$ on $M(g, 1)$ according to

$$
\omega\left(W_{1}, \ldots, W_{3 g-3}\right)=\left\langle\lambda\left|b\left(w_{1}\right) \ldots b\left(w_{3 g-3}\right)\right| \phi\right\rangle
$$

Moreover it is clear using the definition of $|\phi\rangle$ as being parallel transported by $T$, that the variation of the above function in the direction determined by a vector field $V$ can be written as

$$
V\left(\omega\left(W_{1}, \ldots, W_{3 \sigma-3}\right)\right)=\left\langle\lambda\left|b\left(w_{1}\right) \ldots b\left(w_{3 v-3}\right) T(v)\right| \phi\right\rangle
$$


Using this, and $(5,26)$ we can now write $\mu$ as

$$
\begin{gathered}
\mu\left(V_{1}, \ldots, V_{3 g-3+1}\right)=V_{1} \omega\left(V_{2}, \ldots, V_{3 g-3+1}\right)-V_{2} \omega\left(V_{1}, V_{3}, \ldots, V_{3 g-3+1}\right. \\
+\ldots \pm V_{3 g-3+1} \omega\left(V_{1}, V_{2}, \ldots, V_{3 g-3}\right)+\omega\left(\left[V_{1}, V_{2}\right], \ldots, V_{3 g-3+1}\right) \\
+\omega\left(V_{2},\left[V_{1}, V_{3}\right], \ldots, V_{3 g-3+1}\right)+\ldots+\omega\left(V_{2}, V_{3}, \ldots,\left[V_{1}, V_{3 g-3+1}\right]\right) \\
-\omega\left(V_{1},\left[V_{2}, V_{3}\right\}, V_{4}, \ldots, V_{3 g-3+1}\right)-\ldots \pm \omega\left(V_{1}, V_{2}, \ldots,\left[V_{3 g-3}, V_{3 g-3+1}\right]\right) \\
=\partial \omega\left(V_{1}, \ldots, V_{3 g-3+1}\right)
\end{gathered}
$$

where the last equality is a standard mathematical identity. This finally shows that $\mu=\partial \omega$.

\section{Generalizations}

So far we have discussed the simplest conformal theories and one may ask how one could develop an operator formulation for other, more complicated, conformal theories. The list of known conformal theories is growing rather rapidly (see e.g. $\{2][43][44])$. In fact, most of these conformal theories have not been investigated much beyond the tree level and one-loop level. It would be interesting to develop an operator formalism which may aid us in answering questions, such as the question of modular invariance, at higher loops. In this section we briefly comment on how to develop the operator formalism for some of these cases.

We have already described the operator formulation for free bosons, corresponding to compactification on $R^{d}$. It is not much more complicated to describe the case of strings propagating on tori. This can be done along the lines discussed in [9] (see e.g. [45]) or as described in [46]. We have also briefly described the operator formulation on tori with special radius (which is the case of interest for bosonization) in the appendix $A$. The natural direction to proceed from there is the operator formulation for orbifolds which are quotients of $T^{d} / G$, where $G$ is a symmetry of the d-dinuensional torus (for interactions on orbifolds in the path-integral approach see [47]. In fact it turns out that one can describe these in the operator formulation without too much difficulty [48]. The main new ingredient is the existence of the twisted sectors which describe string configurations on tori which are closed only up to the action of some element of the group. We can use the method of conserved charges to characterize interactions involving these states. The only condition is that instead of focusing on harmonic functions, we focus on harmonic 
functions on the surface with appropriate monodromy conditions corresponding to the points of the world sheet where twisted states are emitted. There is a theorem to the effect that we can always find a complete set of conserved charges, which can then be used to characterize the state. In particular this allows us, using the standard technique to construct twist operators: Namely we consider three Hilbert spaces corresponding to twisted sectors $g_{1}, g_{2}, g_{3}$ with $g_{1} g_{2} g_{3}=1$. We characierize the state on the sphere with three punctures at $0, a, \infty$ with coordinates $z, z-a, 1 / z$ and twists $g_{1}, g_{2}, g_{3}$ respectively, by the conserved charge method using holomorphic functions (or vector valued functions in the case of non-abelian orbifolds) with appropriate monodromy. This means that we look at holomorphic functions which transform by $g_{1}, g_{2}$ and $g_{3}$, as we go around 0 , $a$, and

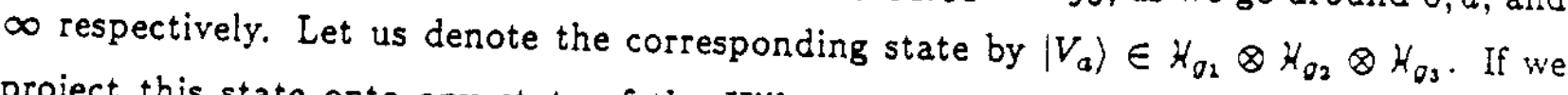
project this state onto any state of the Hilbert space twisted by $g_{2}$, and conjugate the Hilbert space of $\varkappa_{g_{3}},\left|V_{a}\right\rangle$ gives us an operator from $\psi_{\sigma_{3}^{-1}}$ to $\aleph_{g_{3}}$ (where $g_{1}=g_{3}^{-1} g_{2}^{-1}$ ). This operator depends on $a$ and is the vertex operator for emission of twisted state of $\psi_{o}$, which we used to project the state $\left|V_{a}\right\rangle$ at $a$. One can do this also for fermions ard derive the twist operators for fermions (for some previous works on twist operators in the literature see [49]. It is quite satisfactory to see that complicated twist operators which act from one Hilbert space to another, can be easily characterized by the conserved charge method.

We now briefly review the situation for minimal models [2] (we will here mainly focus on the unitary ones [50]). As discussed in [2] the correlation functions on the sphere can be characterized by the differential equations they satisfy. The Feigin-Fuchs method can be used to find solutions to these differential equations [51]. In our formulation the work of $\{2\}$ translates as follows: Consider the state corresponding to a sphere with some number of punctures, where we specify which verma module we put at each puncture (the complete state is the sum over all possible verma modules with the allowed values of $h[2])$. Let us denote this state by $|\phi\rangle$. Degenerate conformal theories have the property that there are states in the verma module which decouple from the physical theory. These are the states which are orthogonal to all the states in the Hilbert space. The condition for their decoupling can be represented as differential equations. The null state can be represented as

$$
|x\rangle=\sum a_{N} \prod L_{-n,}^{k_{1}}|h\rangle
$$


where $N$ collectively labels the indices, and $|h\rangle$ is the primary state with eigenvalue of $L_{0}$ equal to $h$. The differential equation is obtained by setting

$$
\langle\chi \mid \phi\rangle=0
$$

This will be a differential equation for $\langle h \mid \phi\rangle$, as a function of the position of the punctures (here for simplicity we have kept track of the Hilbert space of one puncture only. Properly speaking we should project the state $|\phi\rangle$ onto primary state of all the punctures except for the one we set to $(\chi \mid$.$) . The differential equation is obtained by noting that as discussed$ in section 5 , the action of the Virasoro generators on the state $|\phi\rangle$ can be replaced by differential operators, and in this way the condition (6.1) becomes a differential equation for $\langle h \mid \phi\rangle$. Of course this does not completely finish the task of constructing the conformal theory on the sphere, because the differential equation has more than one solution, and one has to know how to combine the solutions for the left-movers with those of the rightmovers to obtain modular invariant combinations. Even though this can be done $[51]$, one would like a more general way of doing it.

One is naturally inclined to ask what happens at higher genus. This question has been considered in some works in the literature [52]. More recently it has been discovered that the partition functions of the discrete series can be classified by solutions of a single differential equation, by considering torus with a single puncture [53][54]. In general one obtains [54] overdetermined differential equations which one would therefore naively expect to have no solutions, but turn out to have solutions! This already happens at the level of the sphere with three punctures (corresponding to the OPE), where one ends up with more polynomial equations than unknowns (the $h$ 's) but one ends up with solutions precisely at the values of $h$ determined by the Kac formula. Similar things happen at higher genus. For example the null state for $c=1 / 2$ for the vacuum Verma module at genus 2 with one puncture results in 11 differential equations in three unknowns. So apriori one expects no solutions. But magically one obtains solutions. This magic still remains to be understood.

A similar story repeats for the Kac-Moody algebras at higher genus. In these cases we can use the null states of the verma module of the combined Virasoro algebra and the Kac-Moody algebra, to obtain differential equations. Again, by considering the torus with one puncture, one can obtain [55]|54] differential equations for the partition functions of the representations of Kac-Moody algebras (including the twist partition functions with the Cartan subalgebra). Again the system is overdetermined and only unexpectedly one ends up with solutions. 
One may ask why we do not simply sew spheres together to obtain higher genus amplitudes. In fact we can. But to prove issues such as modular invariance we have to find an apriori way of characterizing the states that we get for higher genus. Once we know this, we can use the analog of CTA argument to justify the sewing of states to obtain the higher genus states.

It is clear that a lct more work remains to be done for a better understanding of the operator formalism for complicated conformal theories, such as $c<1$ theories.

\section{Conclusion}

In this paper we have considered the operator formulation for conformal theories, and in particular, we have given a detailed description of some simple ones, including the Polyakov strings in fiat background spaces.

We have seen how Virasoro algebra can be used to move the states on the moduli space of surfaces, i.e., giving us parallel transport. In particular we have seen that issues such as modular invariance can be addressed without undue difficulty completely in the operator language. Moreover the operator language combines all the scattering amplitudes (for a fixed number of particles) into one master state. In this sense it is more natural than the path-integral approach.

Most of what we have described in this paper is not surprising. We expected there to be an operator formulation for conformal theories. The main thing to emphasize, however, is that we have seen that the operator formulation not only does not have any disadvantages compared to path-integral approach, but that the main properties of the theory such as factorization and other properties of the the partition function are automatic in this formulation. Moreover it is not known if all the conformal theories can be described by a path-integral, whereas any path-integral formulation, has an operator content.

More work remains to be done: We have been describing the closed bosonic conformal theories in this paper. For superstrings we have to extend all this formalism, where we know the situation is going to be more complicated due to the presence of super-ghosts. Recent work shows [56] that there are ambiguities in the integration over supermoduli space that represents the superstring amplitudes in perturbation theory. It would be interesting to look at superstrings from the operator point of view in connection with this ambiguity. It is clear from the discussions of sections 4,5 that the analysis of infinities, unitarity and factorization are more straightforward in the operator approach. As shown 
in [3] the most efficient way of dealing with the spinor ghosts and picture changing operations that appear in superstring theories is in the operator formalism. The operator approach that may be developed for superstrings along the lines discussed in this paper [57] may be a compromise between the tree level arguments contained in that work, and the functional integral approach used in the explicit construction of super and heterotic strings $[56]$. Also so far we have described some of the simplest examples of conformal theories (toroidal compactification, orbifolds). It would be interesting to do some more complicated examples, such as the minimal models, in detail. In particular, the behaviour of these models in higher genus surfaces remains largely unexplored. Further progress on this direction seems to require a more geometrical understanding of the Feigin-Fuchs construction of the correlation functions on punctured spheres. A deeper knowledge of the interplay of the monodromy conditions and modular invariance in higher genus for the minimal series is still lacking. It is clear that the basic setup we have been presenting (for example the relation of Virasoro algebra with motion in moduli space) is common to all conformal theories. It seems likely that methods similar to those described here will help in obtaining answers to these questions, and will also provide efficient tools to explore the geometry of conformal field theories.

We are now at a stage in the development of string theories where we know how to compute scattering amplitudes in some simple backgrounds. Many technical issues still remain unresolved regarding finiteness and unitarity questions even in the simplest vacuum configurations. Equally important is the understanding of the geometry, both local and global, of the space of all conformal field theories. We have not yet achieved any deep understanding of the intrinsic kinematical properties of string theory, and virtually nothing is known about the dynamics that selects background spacetimes out of the plethora of conformal field theories. Some important pieces in this puzzle are still missing.

We would like to thank C. Reina for collaboration on early stages of this work. We would also like to thank E. Arbarello, F. Bien, S. Giddings, D. Kazhdan, E. Martinec, P. Nelson, D. Olive, G. Segal and E. Witten for discussions.

Th research of L.A. was supported by DOE contract DE-AC02-88ER-40284, of G.M1. by DOE contract DE-AC02-76ER-02220, of C.V. in part by NSF contract PHY-82-15249 and by a fellowship from Harvard Society of Fellows. 
Appendix A. Grassmannians and the Algebraic Proof of Bosonization

This appendix can be read independently of the rest of this paper. It relies heavily on the work of Segal and Wilson $|16|$, but we will review the necessary parts of that paper where appropriate. What we would like to do is to give a purely algebraic proof of chiral bosonization on arbitrary Riemann surfaces (for path-integral approach see $[20][21][22][23][58])$.

We begin with a review of some of the more mathematical definitions involved in the theory of Grassmannians. For a more thorough treatment see [16]. The grassmannian of a Hilbert space $H: \operatorname{Gr}(H)$ or simply $\mathrm{Gr}$ is defined as follows : First we split $H$ into two orthogonal subspaces $H=H_{+}+H_{-}$. In the case at hand, $H$ is the space of first quantized wave functions on the circle, $H_{+}$is the space of negative energy states, and $H_{-}$is the subspace of positive energy states. Then $\mathrm{Gr}$ is the collection of all subspaces $W \subset H$ satisfying

1). The orthogonal projection pr $: W \rightarrow H_{+}$is a Fredholm operator .

2). The orthogonal projection pr $: W \rightarrow H_{-}$is a Hilbert-Schmidt operator.

Recall that a Hilbert-Schmidt operator $T$ on a Hilbert space $H$ has the property that $\sum\left\|T e_{i}\right\|^{2}<\infty$ for any orthonormal basis $e_{i}$ of $H$.

Since $p r_{+}$is a Fredholm operator, its index is an integer, and $\mathrm{Gr}$ has an infinite number of disconnected components labelled by ind $\mathrm{pr}_{+}$. There is a covering of $\mathrm{Gr}_{\mathrm{r}}$ by open patches related to the free fermionic Fock space. Given one of the $H_{S}$ 's we define the subset $U_{S}$ as the collection of all $W \in G r$ such that the orthogonal projection pr : $W \rightarrow H_{S}$ is an isomorphism. A coordinate system for the patch $U_{S}$ is obtained by giving for each $W \in U_{S}$ a Hilbert-Schmidt operator $B(W): W \rightarrow W^{\perp}$. Thus if $H_{S}$ has a basis $z^{n_{1}}, z^{n_{2}}, \ldots ; n_{1}<n_{2}<\ldots$, the functions

$$
w_{n_{i}}=z^{-n_{i}}+\sum_{z^{m} \in H_{s}} B_{n_{1} m} z^{m}
$$

give a basis for $\mathrm{W}$. In particular, for $H_{S}=H_{+}$the tangent space to $\mathrm{Gr}$ at any point is isomorphic to the space of Hilbert-Schmidt operators $B: H_{+} \rightarrow H_{-}$. We may think of the $B_{n m}$ as local coordinates on $G r$.

The space $G r$ has several interesting and useful properties, some of which we now briefly explore. $G r$ is an infinite dimensional homogeneous space. There is a group $G L_{r c}$, which acts transitively on Gr. The group $G L(H)$ of all invertible operators on $H$ is far too big to preserve conditions 1) and 2). One can nevertheless consider the largest subgroup 
of $G L(H)$ which acts on Gr. Decomposing the elements of $G L(H)$ with respect to the splitting we have chosen of $H$, any element $g \in G L(H)$ can be expressed as $g=\left(\begin{array}{ll}a & b \\ c & d\end{array}\right)$ , and $\mathbf{g}$ will act on $\mathrm{Gr}$ if and only if $\mathrm{a}$ and $\mathrm{d}$ are Fredholm, and $b, c$ are Hilbert-Schmidt - These properties are easily seen to be preserved under multiplication. The subgroup of $G L(H)$ satisfying these conditions is called $G L_{r e s}$. It is not difficult to prove that $G L_{\text {res }}$ and its subgroup $U_{\text {ree }}$ of unitary operators act transitively on Gr. If $N_{+}$is the group of matrices satisfying $c=0$, we can represent $\mathrm{Gr}$ as

$$
G r=\frac{G L_{\text {res }}}{N_{+}}=\frac{U_{\text {res }}}{U\left(H_{+}\right) \times U\left(H_{-}\right)}
$$

where $N_{+}$is the isotropy subgroup of $H_{+}$in $G L_{\text {ree }}$ and $U\left(H_{+}\right) \times U\left(H_{-}\right)$is the isotropy subgroup of $H_{+}$in $U_{\text {res }}$.

Using all this information, it is easy to construct the coordinate transformations between two overlapping patches $U_{W_{1}}, U_{W_{2}}$ defined with respect to two points $W_{1}, W_{2}$ Since we will not need these formulae, we refer the interested reader to the literature [16].

We can associate a ray in the fermionic Fock space to any point $W$ in Gr. Geometrically this corresponds to constructing a line bundle over $\mathrm{Gr}$.For the finite dimensional grassmannian of $k$-planes in $C^{n}: G r_{k, n}$ the determinant line bundle is the top exterior power of the k-plane labelled by each point in $G r_{k, n}$. In infinite dimensions we have to be more careful. Given $W \in G r$ the determinant does not exist for any basis of $W$. A basis for $\mathrm{W}$ is an operator $w: H_{+} \rightarrow H$ whose restriction to $H_{+}, w_{+}$is Fredholm and whose restriction to $H_{-}$is Hilbert-Schmidt. A basis is admissible, if $w_{+}$differs from the identity by an operator of trace class. In particular, $w_{+}$has a determinant [59].

If $P$ is the space of admissible bases, $P$ can be thought of as a vector bundle over Gr. The fiber group $T$ is right multiplication by $t$ with $t \in G L\left(H_{+}\right)$of the form $1+$ trace class. The grassmannian can be expressed as $P / T$ and the determinant line bundle $D E T=P \times_{T} C$ with the equivalence relation $(w, \lambda) \sim\left(w t^{-1}, \lambda\right.$ det $\left.t\right)$, for any admissible basis and complex number $\lambda$.The dual of the determinant bundle $D E T^{-1}$ is defined with the equivalence relation $(w, \lambda) \sim(w t, \lambda$ det $t)$. From the path integral description of sections of $D E T^{-1}$ (see section two) together with the general principal of conformal field theory that states on a circle are equivalent to operator insertions in a disk one can show that the free fermionic Fock space is naturally identified with $\Gamma\left(D E T^{-1}\right)$ [11 $\left.\mid 16\right]$.

Next we exhibit how the state $|B(W)\rangle$ of section two, constructed from an element $W \in G r$ contains the information about a geometrical quartet of data $m=(\Sigma, L, P, z)$, 
where $\Sigma$ is a Riemann surface, $L$ is a line bundle over $\Sigma, P$ is a point in $\Sigma$ and $z$ is a local coordinate around $P$ with $z(P)=\infty$. We first show how this geometrical data gives a point in Gr. Let $M_{0}$ be the moduli space of Riemann surfaces of genus $g$, (for definitions and references, see for example [15]), and let $P_{\theta}$ be the moduli space of the geometrical data described above. The space $H^{\circ}(\Sigma-P, L)$ is the space of holomorphic sections of $\mathrm{L}$ over $\Sigma-P$ thus $\psi \in H^{0}(\Sigma-P, L)$ can have poles of arbitrary order at $\mathrm{P}$. The Krichever map $k: P_{\mathfrak{g}} \rightarrow \operatorname{Gr}[60]$ is defined to be $k(m)=H^{0}(\Sigma-P, L)$. To understand why this map gives a point in $\mathrm{Gr}$, we cover the surface with two coordinate patches $U_{0}, U_{\infty}$ where $U_{0}$ is the surface minus the point $P$, and $U_{\infty}$ is a disc around $P$ where the local coordinate is defined. The overlap region is an annulus $A$ centered upon $\mathrm{P}$, and this is a good covering to describe many of the complex properties of the Riemann surface. The map pr+acting on any holomorphic section of $\mathrm{L}$ over $\mathbf{A}$ gives its polar part. This is an element in $H_{+}$. The global holomorphic sections of $\mathrm{L}$ over $\Sigma: H^{0}(\Sigma, L)$ coincide with the global solutions of the equation $\bar{\partial} \psi=0$, where $\psi$ is a section of $L$. The holomorphic sections of $L$ over $A$ which cannot be written as $\psi_{0}+\psi_{\infty}$ where $\psi_{0}$ is a holomorphic section over $\Sigma-P$ and $\psi_{\infty}$ is holomorphic over $U_{\infty}$ is called $H^{2}(\Sigma, L)$ in the mathematics literature [17], and it can be shown to be isomorphic to the cokernel of the operator $\bar{\partial}$ acting on sections of $L$. It is also dual to the space of holomorphic sections of $(1,0)$ forms on $\Sigma$ with values in $L^{-1}$ , the line bundle whose transition functions are the inverse of those defining $\mathrm{L}$. We have an exact sequence

$$
0 \rightarrow H^{0}(\Sigma, L) \rightarrow W \rightarrow H_{+} \rightarrow H^{1}(\Sigma, L) \rightarrow 0
$$

the first map is inclusion, the second is $p r+$ and the last gives the Laurent tails of sections of those elements of $W$ which cannot be written as a sum of holomorphic sections over $U_{0}$ and $U_{\infty}$. The exactness of (A.3) implies that $\mathrm{Kerpr}_{+}=\operatorname{ker}_{L}$ and $\mathrm{Cokpr}_{+}=\mathrm{Cok}_{L_{L}}$. Since these spaces are finite dimensional, $p r+$ is Fredholm .

Using the Krichever map we pull-back the bundle $D E T^{-1}$ to obtain a line bundle over $P_{0}$. Equation (A.3) implies that the pull-back bundle is isomorphic to the determinant line bundle of the Cauchy-Riemann operator acting on sections of $\mathrm{L}: D E T \overline{\partial_{L}} \equiv \wedge H^{0}(\Sigma, L) \otimes$ $\left(\bigwedge H^{1}(\Sigma, L)\right)^{-1}$ where $\bigwedge$ stands for the highest exterior power of a vector space. Since the kernel and cokernel of the Cauchy-Riemann operator do not depend on the local coordinate or the position of the point $\mathrm{P}$, we obtain a line bundle over $M_{0}$, the moduli space of curves The results of previous paragraphs guarantee that we can associate a vacuum state for a fermionic Fock space to the geometrical data in each point of $P_{v}$. As an example let $L$ be 
a twisted spinor on $\Sigma$. The point $W$ in $G$ r obtained by the Krichever map will be in $U_{H}$. if $\operatorname{Ker} \overline{\partial_{L}}=\operatorname{Cok} \overline{\partial_{L}}=0$.

The starting point for the proof of bosonization will be the explicit expression for the state $|B(W)\rangle$ derived in the section two (2.18), and the construction of the state associated to a multivalued scalar. Since we are always working with field theory on an annulus, we can use rany of the standard bosonization formulae that hold in the simpler case of fermions moving on the complex plane. The algebraic proof of bosonization can be thought of as a consequence of the vertex operator representation for the $U(1)$-current algebra [61] [62]

We now describe the appropriate coordinates for bosonic wavefunctions. A natural subgroup of $G L_{\text {ree }}$ is the group $\Gamma$ of non-vanishing complex valued functions on the circle - The action of $\Gamma$ on the Fock space is given by the fermion number current. Two distinguished subgroups of $\Gamma$ are $\Gamma_{+}$and $\Gamma_{-} ; \Gamma_{+}$is the subset of $\Gamma$ of boundary values of holomorphic functions on the disc $D_{0}=\{z \in C,|z| \leq 1\}$, and $\Gamma_{-}$is the subset of boundary values of holomorphic functions on the disc $D_{\infty}=\{z \in C,|z| \geq 1\}$. These functions have zero winding number. The functions $z^{n}$ for any $n$ have winding number $n$ and they are also in $\Gamma$.

Any element of $\Gamma_{+}$(resp. $\left.\Gamma_{-}\right)$can be written as $e^{f}$ (resp. $e^{j}$ ) with $f(z)=$ $\sum_{n>0} x_{n} z^{n}$ (resp. $f(z)=\sum_{n>0} \tilde{x}_{n} z^{-n}$ ). These group elements act on the Fock space via the operators

$$
\begin{gathered}
e^{H(x)} \text { where } H(x)=\sum_{n>0} x_{n} j_{n}=\frac{1}{2 \pi i} \oint_{p} j(z) f(z) \\
e^{H(z)} \quad H(\bar{x})=\sum_{n>0} \bar{x}_{n j-n}=\frac{1}{2 \pi i} \oint_{p} j(z) \tilde{f}(z)
\end{gathered}
$$

where $j_{n}$ was defined in (2.51). From (2.8) we obtain

$$
e^{H(x)} e^{H(x)} e^{-H(x)} e^{-H(x)}=e^{-S(j, f)}
$$

with

$$
S(\tilde{f}, f)=\frac{1}{2 \pi i} \oint_{P} d \tilde{f} f=-\sum_{n>0} n I_{n} \tilde{I}_{n}
$$

We can write the current algebra in the Schrödinger representation as in (2.55). Any state in the fermionic Fock space can be represented in terms of functions $\psi\left(I_{1}, I_{2}, \ldots\right)$. 
Since $j_{-n}^{\dagger}=j_{n}$, we want to find a scalar product in the Schrödinger representation which satisfies this hermiticity condition. A simple computation shows that $63 j$ this scalar product is given by

$$
\begin{gathered}
\left\langle x_{1}^{n_{1}} \ldots x_{k}^{n_{k}} \mid x_{1}^{n_{1}} \ldots x_{k}^{n_{k}}\right\rangle=\frac{n_{1} ! \ldots n_{k} !}{1^{n_{1}} 2^{n_{2}} \ldots k^{n_{k}}} \\
\langle f(x) \mid g(x)\rangle=\left.f\left(\frac{\partial}{\partial x_{1}}, \ldots, \frac{\partial}{k \partial x_{k}} \ldots\right) g(x)\right|_{x=0}
\end{gathered}
$$

this bracket is known in the soliton literature as the Hirota bilinear operation [63].

The standard bosonization amounts to associating to any state in the fermionic Fock space a wave function. Since we are working on a disc, we can use the standard rules to express the field operators $b(z), c(z)$ in terms of the fermion current. This representation depends on the charge sector of the Fock space. Since fermion number $Q$ is conserved, we can split the Fock space into sectors with different charge $F=\oplus F_{m}, Q F_{m}=m F_{m}$ - The vacuum in $F_{0}$ is the standard Dirac vacuum $(2.11)$ while the ground state $|m\rangle$ in $F_{m}$ is obtained from $|0\rangle$ by shifting the Dirac sea up to level $\mathrm{m}$. The fermionic two point function in any sector is

$$
\langle m|c(z) b(w)| m\rangle=\left(\frac{w}{z}\right)^{m} \frac{1}{(z-w)}
$$

if we represent the vacuum $|m\rangle$ by the function $1, c(z)$ and $b(z)$ become $[64][65][63 !$

$$
\begin{gathered}
c(z)=V(x, z)=z^{-m}: e^{i X(z)}: \\
b(z)=V^{*}(x, z)=z^{m}: e^{-i X(z)}: \\
X(z)=q-i p \ln z+i \sum_{n \neq 0} j_{n} \frac{z^{-n}}{n}
\end{gathered}
$$

after some algebra one obtains

$$
\frac{\left\langle m\left|\prod_{i} c\left(z_{i}\right) b\left(w_{i}\right)\right| B, m\right\rangle}{\langle m \mid B, m\rangle}=\left.\frac{\prod_{i} V\left(z_{i}\right) V^{*}\left(w_{i}\right)\left\langle m\left|e^{H(x)}\right| B, m\right\rangle}{\langle m \mid B, m\rangle}\right|_{x=0}
$$

the function 


$$
\frac{\left\langle m\left|e^{H(x)}\right| B, m\right\rangle}{\langle m \mid B, m\rangle}=\tau_{B}(f)
$$

is the wave function associated to the state $|B, m\rangle$, and it is also known as the $\tau$ function in soliton theory $[16],[66]$. To obtain an explicit bosonic representation of the fermionic correlation functions, we have to compute $r(f)$ explicitly in terms of the $x_{n}$ variables .

The computation of $\tau$ is based on its behavior with respect to the group $\Gamma_{+}$. Using the covering of the surface $\Sigma$ introduced in the previous section, let $V$ be the space of functions such that $e^{V}=\Gamma$. The geometrical action of $\Gamma_{+}$on the Krichever data, is to change the line bundle $L$ by tensoring a flat line bundle whose transition function in $U_{0} \cap U_{\infty}$ is an element $g \in \Gamma_{+}$. The space of flat line bundles is just the zeroth component of $H^{1}\left(\Sigma, O^{*}\right)_{0} \cong H^{1}(\Sigma, O) / H^{1}(\Sigma, Z)^{10}$, i.e. the Jacobian of the Riemann surface, and in particular is finite dimensional. Hence the mapping from $V$ to $H^{1}(\Sigma, O)$ has a huge kernel $K_{0}$ given by those functions $f \in V$ which can be written as $k=k_{0}+k_{\infty}$ where $k_{0}$ is a function which extends to a holomorphic function in $U_{0}$ and $k_{\infty}$ extends holomorphically to $U_{\infty}$. Since the fermionic two point function involves theta functions, we should expect that the $\tau$-function should be defined in the jacobian of the surface. We can construct a mapping $V \rightarrow J(\Sigma)$ whose kernel $K$ is such that $K / K_{0}=H^{1}(\Sigma, Z)$. In other words, if $k \in K$, we can write $e^{k}=e^{(\phi(k))} e^{k_{\infty}}$ where the function $\phi(k)$ is holomorphic off $\mathrm{P}$ and multivalued with shifts $2 \pi i Z$ around the homology. One can construct the functions $\phi(k)$ explicitly using the differentials of the second kind (2.47) and the meromorphic differentials that can be associated to a given homology cycle. If we consider the space of multivalued functions holomorphic in $\Sigma-P$ whose exterior derivative is single valued, then we are effectively dealing with the space of differentials of the second kind with poles at $P$ : $H^{0}(\Sigma-P, K)$, with $K$ the canonical line bundle. One can show [67] that to any curve $\gamma$ one can associate a meromorphic differential $\nu(\gamma)$ and thus a multivalued function $\eta(\gamma)$ so that the integral of any meromorphic form times $\eta(\gamma)$ aruund $P$ gives the line integral of the meromorphic differential around the cycle $\gamma$. For a canonical homology basis, we can construct th en multivalued functions possibly with poles at $\boldsymbol{P}$ such that they represent the cycles $a_{i}, b_{i}$. We will denote these functions by $\eta_{i}, \eta_{i+g}$ for $i=1, \ldots g$, where the first $\mathrm{g}$ are associated to the $a$-cycles and the last $\mathrm{g}$ to the b-cycles. To define the mapping

$10 O$ is the structure sheaf, i.e. the sheaf of holomorphic functions on the surface. 
from $V \rightarrow H^{1}(\Sigma, O)$ we need to find a function which for vanishes for every $k \in K$. This is given by $f \rightarrow \bar{f}$ where

$$
\bar{f}=\frac{1}{2 \pi i} \oint_{P} f(z) \omega(z)
$$

this clearly vanishes for elements of $K_{0}$ because the abelian differentials are holomorphic globally, and $k$ can be split into a function holomorphic in $U_{0}$ plus a function holomorphic in $U_{\infty}$. Thus we can deform the contour in (A.14) towards P or to the back of the surface to obtain 0 . For functions $k \in K$, this map will give the jacobian lattice $\tau Z^{g}+Z^{0}$ where $\tau$ is the period matrix for the choice of marking. Thus

$$
\bar{k}=\tau a+h
$$

and $a, b$ are $g$-dimensional vectors with integral entries. Given one such function $k$, the function $\phi(k)$ is

$$
\begin{gathered}
\phi(k)=\int^{t}\left(\eta_{n}(t) y_{n}+2 \pi i \omega(t) \cdot a\right) \\
k(t)=\sum_{n \geq 1} y_{n} t^{-n}
\end{gathered}
$$

similarly, using the $\eta_{n}$-differentials we can construct functions in $H^{0}(\Sigma-P, O)$

$$
g_{n}(t)=\int^{t} \eta_{n}(t)-2 \pi i A_{n}^{i} \eta_{i}
$$

the $\eta_{n}(t)$ differentials have zero a-periods, and their b-periods are given by

$$
\oint_{b_{i}} \eta_{n}(t)=-2 \pi i A_{n}
$$

and $A_{n}$ is defined in (2.46). The first simple property that one can derive of the state $|B\rangle$ is that it is annihilated by the charges

$$
Q\left(g_{n}\right)=\oint_{P} j(t) g_{n}(t) \quad Q\left(g_{n}\right)|B\rangle=0
$$

this is because the current $j(z)$ acting on the state $|b\rangle$ behaves like a meromorphic differential on the surface with poles only at $P$. This follows from the explicit form of the expectation value of the fermionic current between the state $|B\rangle$ and any state in the free 
fermionic Fock space. Thus the state associated to the fermions on the surface is invariant under the action of $K_{0}$. This is not so for all of $K$. Nevertheless, we can derive the transformation properties of the $\tau$-function with respect to $K$. First of all we note that the commutation relations between the charges (A.19) for functions in $K$ are

$$
\left[Q\left(\phi_{1}\right), Q\left(\phi_{2}\right)\right]=2 \pi i\left(a_{1} \cdot b_{2}-a_{2} \cdot b_{1}\right)
$$

hence we get a realization of the intersection pairing on the surface in terms if the operators implementing the action of $K$ in the Fock space (see [46]). In particular (A.20) implies that

$$
e^{Q\left(\phi_{1}\right)} e^{Q\left(\phi_{2}\right)}=e^{Q\left(\phi_{2}\right)} e^{Q\left(\phi_{1}\right)}
$$

to compute the change of the $\tau$-function under $K$ we want to evaluate

$$
\left\langle 0\left|e^{\oint j(t) k(t)} e^{\oint j(t) f(t, x)}\right| B\right\rangle=\tau(f+k)
$$

since the two operators that appear in (A.22) are in $\Gamma_{+}$, they commute. Next, we split $k(t)$ into $k_{0}+k_{\infty}$. Since the components $j_{n}, n>0$ annihilate the vacuum on the right, the components $j_{-n}$ annihilate the vacuum on the left. Using (3.1-3) we obtain

$$
\begin{gathered}
\tau(f+k)=\left\langle 0\left|e^{\oint j(t) f(t, x)} e^{\oint j(t) k_{0}}\right| B\right\rangle e^{t\left|j\left(k_{0}\right), j\left(k_{\infty}\right)\right|+S\left(k_{\infty}, f\right)}= \\
\tau(f) \tau(k) e^{S\left(k_{\infty}, f\right)}
\end{gathered}
$$

In the last step we use the fact that the charges corresponding to elements on $K_{0}$ annihilate $|B\rangle$ to show that $\exp \oint j(t) k_{0}|B\rangle=$ const. $|B\rangle$. When $f$ is equal to zero, the first line of (A.23) gives the $r$-function with argument $k$. It is now easy to solve (A.23) using the explicit form of the functions $\phi(k)$. The cocycle factor $S\left(k_{\infty}, f\right)$ can be written as

$$
S\left(k_{\infty}, f\right)=\sum_{n m \geq 1} Q_{n m} y_{n} I_{m}-2 \pi A_{n} \cdot a
$$

where the coefficients $Q_{n m}$ are defined in (2.48). Define the quadratic form

$$
Q(f, f)=Q_{n m} x_{n} x_{m}
$$


then for functions $k \in K_{0} S\left(k_{\infty}, f\right)=2 Q(k, f)$. Defining the new function $F(f)$ by

$$
\tau(f)=e^{Q(f, f)} F(f)
$$

we obtain for any two elements in $K_{0}$

$$
F\left(k_{1}+k_{2}\right)=F\left(k_{1}\right) F\left(k_{2}\right)
$$

hence the logarithm of the $\tau$-function is a linear function on $K_{0}$. Let us extend this function by linearity to the whole of $\mathrm{V}$, and call it $\alpha(f)$. Then the function

$$
\tilde{F}(f)=F(f) e^{-\alpha(f)}
$$

is defined only on $K / K_{0} ;$ using (A.24) we get

$$
\tilde{F}(f+k)=\tilde{F}(f) \tilde{F}(k) e^{-2 \pi i A_{n} x_{n} \cdot a}
$$

From the definition we gave of the $\tau$-function, $\tau(0)=0$. Using this plus the equivalent form of the previous equation

$$
\bar{F}(\bar{f}+\tau a+b)=\bar{F}(\bar{f}) \tilde{F}(\tau a+b) e^{-2 \pi \bar{f} \cdot a}
$$

we can write $\tilde{F}$ in terms of theta functions [18]

$$
\tilde{F}(\bar{f})=\frac{\theta\left(\begin{array}{l}
\alpha \\
\beta
\end{array}\right)(\bar{f})}{\theta\left(\begin{array}{l}
\alpha \\
\beta
\end{array}\right)(0 \mid r)}
$$

the function $\alpha(f)$ is determined now by looking at the two point function for the fermions. In this case we get $\alpha(f)=0$ and we can finally write down the explicit form of the $r$-function for spin $1 / 2$ fermions as

$$
r(x)=e^{Q_{n m} x_{a x m}} \frac{\theta\left(\begin{array}{l}
\alpha \\
\beta
\end{array}\right)\left(A_{n} x_{n} \mid \tau\right)}{\theta\left(\begin{array}{l}
\alpha \\
\beta
\end{array}\right)(0 \mid \tau)}
$$

For a $b-c$ system of spin $j$, we know from the Riemann-Roch theorem that the sections of $K^{1-j}$ holomorphic off $\mathbf{P}$ must have poles of order at least $q+1$ with $q=(2 j-1)(g-1)$ 
- From the Krichever map, the point obtained in the grassmannian sits in the component of index $-q$. We can absorb all the vacuum charge at the point $P$, and then the point $W$ in the grassmannian is obtained by computing the Szego kernel for the $b-c$ system $\langle c(z) b(w)\rangle$ with a pole of order $q$ at $P$ in $z$, and a zero of order $q$ at $P$ in $w$. Since the two point function also has a pole of first order as $z \rightarrow w$, these conditions uniquely determine the two doint function to be

with

$$
S(t, y ; j)=\left(\frac{E(t, P)}{E(y, P)}\right)^{q}\left(\frac{\sigma(y)}{\sigma(t)}\right)^{2 j-1} \frac{1}{E(t, y)} \frac{\theta\left(\int_{y}^{t}+e i \tau\right)}{\theta\left(e^{i} \tau\right)}
$$

$$
e=I\left(K^{j}(-q P)\right) \otimes L_{\Delta}^{-1}
$$

the image under the Jacobi map $[18]$ of the line bundle $K^{j}(-q P) \otimes L_{\Delta}^{-1}$ and $\Delta$ is the Riemann class associated to the marking chosen on $\Sigma$. The function $\sigma(t)$ is a $g / 2$ differential defined on the covering of the Riemann surface. It has no zeroes or poles, is periodic around the a-cycles and multivalued around the b-cycles [18] with period:

$$
\sigma\left(t+n_{i} \cdot b_{i}\right)=\sigma(t) \exp \left(-i \pi(g-1) n \cdot \tau \cdot n+2 \pi i \int_{(g-1) P}^{\Delta} \omega \cdot n\right)
$$

thus in this case, using the vertex operators (3.7) with $m=-q$ we obtain again a $\tau$-function

and $\alpha(f)$ is determined by

$$
\tau\left(x_{n}\right)=e^{Q_{n m} x_{n} x_{m}+\alpha_{n} x_{n}} \frac{\theta\left(A_{n} x_{n}+e \mid \tau\right)}{\theta(0 \mid \tau)}
$$

$$
e^{-\sum \alpha_{n} \frac{2 \pi}{n}}=\left(\frac{E(t, P)}{t}\right)^{q}(\sigma(t))^{2 j-1}
$$

Let us now come to the case of a multivalued scalar on the surface. We want to show that with appropriate choices for the twistings, the corresponding $\tau$-function coincides with (A.27). There are at least two ways to carry out the operator formulation. One is based.on a hybrid between functional integral and operator arguments [9]. Since we know that in this case one has to include instanton configurations in the functional integrals , the state one gets for a given instanton sector should give an expectation value to the scalar field equal to the instanton solution. This completely determines the modification 
one has to make on the state associated to a single-valued scalar in terms of a coherent state (as well as multiplying the normalization by the instanton action). The complete state one then obtains is a sum over the states for each instanton sector. There is however a different way [46] of obtaining the same result which only uses operator methods, which we will follow.

We follow the same procedure as with the single-valued scalars. Since singlevaluedness is not an issue any more, we can consider shifting the field $X$ by multivalued holomorphic functions off $\mathrm{P}$. We split the field $X$ into its holomorphic and antiholomorphic oscillators, and carry out the explicit computations for the holomorphic part only. The arguments for the antiholomorphic part are entirely similar. If $f \in H^{0}(\Sigma-P, O), f+\bar{f}$ is a harmonic function and we obtain the conserved charges

$$
Q(f)=\oint_{P} f(t) \partial X(t)
$$

where from now on the contour integral includes the factor of $\frac{1}{2 \pi i}$. As pointed out in the previous section, these charges are not enough to construct the state for a scalar on $\Sigma$ - Now however we have a richer set of functions. For multivalued functions holomorphic off $P$ the charges (3.14) do not all commute. Their commutator is related to their shifts around the homology (3.15) and this means that we cannot consistently require that all such functions annihilate the state $|\Phi\rangle$ for the twisted scalar. Since the commutator of the charges associated to single valued functions commute among themselves and with those obtained for multivalued functions, we can choose $\mathrm{g}$ commuting charges out of those obtained using the functions $\nu_{i}, \nu_{i+g}$, defined above, and require that the state $|\Phi\rangle$ be an eigenfunction of these oscillators. The spectrum of eigenvalues is determined by the twisting of the scalar feld, and they form a lattice that corresponds to the lattice used to define the circle where the scalar field moves times the jacobian lattice for the surface. The eigenvalues represent the flow of momentum of the scalar field through the corresponding homology cycles. We thus have the following conditions for the state $|\Phi\rangle$

$$
\begin{gathered}
Q(f)|\Phi, k\rangle=0, f \in H^{\circ}(\Sigma-P, O) \\
Q\left(a_{i}\right)|\Phi, k\rangle=k_{i}|\Phi, k\rangle \\
Q\left(a_{i}\right)=\oint j(t) \nu_{i} \quad, i=1, \ldots g
\end{gathered}
$$


in terms of the Schrödinger representation of the $U(1)$-current algebra, these equations can be written explicitly using (A.17) and (2.50) as

$$
\begin{gathered}
\left(\frac{\partial}{\partial x_{n}}-2 Q_{n m} x_{m}-2 \pi i A_{n}^{i} Q\left(a_{i}\right)\right)|\Phi, k\rangle=0 \\
Q\left(a_{i}\right)|\Phi, k\rangle=k_{i}|\phi, k\rangle
\end{gathered}
$$

the solution of these equations involves an arbitrary function of the momentum $k$ :

$$
|\Phi, k\rangle=e^{Q_{n m} x_{n} x_{m}+2 \pi i A_{n}^{i} x_{n} k_{i}+r(k)}
$$

to determine the explicit form of the function $r(k)$ we simply note that the operators $Q(\phi(k))$ (A.16) commute with $\exp Q(f)$ and shift the value of the momentum $k \rightarrow k+a$ $(3.11,15)$. We have to consider the exponentiated operator, because the momentum is quantized, and only finite translations are defined on the Fock space. This operator changes the state $|\Phi, k\rangle$ to $|\Phi, k+a\rangle$. Thus we obtain

$$
e^{Q(\phi(k))}|\Phi, k\rangle=\text { const. }|\Phi, k+a\rangle
$$

using (A.16) and the explicit Laurent expansion of the differentials $\theta_{n}$ in terms of $Q_{n m}$ 's , one easily obtains that

$$
|\Phi, k\rangle=\exp \left(Q_{n m} x_{n} x_{m}+i \pi k \cdot \tau \cdot k+2 \pi i k \cdot\left(A_{n} x_{n}+\beta\right)\right)
$$

the full state is now obtained by summing over the allowed values of the momentum $k$ which depends on the explicit compactification considered .If we write $k=\alpha+Z^{j}$ with $\alpha$ a 8 -dimensional vector, the final answer is

$$
|\Phi\rangle=e^{Q_{n} m x_{n} x_{m}} \sum_{k \in Z^{g}+a} e^{i \pi k+k+2 \pi i k \cdot\left(A_{n} x_{n}+B\right)}
$$

which up to a constant coincides with (A.27). This concludes our algebraic proof of the bosonization formulae. The case of toroidal compactification with an arbitrary radius has been discussed in $[46][45]$. 


\section{Appencix B. The Weierstrass gap theorem}

At several points we have had occasion to use an application of the Riemann-Roch theorem known as the Weierstrass gap theorem. For the convenience of the reader we give the statement and proof of this theorem below.

Theorem 1: At a generic point $p \in \Sigma$ on a Riemann surface consider the space of sections of $\omega^{j}$ and $\omega^{1-j}$ for $j \geq 3 / 2$ which are meromorphic at $p$ and holomorphic elsewhere. Denote these spaces $B^{j}(\tau, p)$. In a local coordinate $t$ at $p$ we can write bases for $B^{j}$ and $B^{1-j}$ which look like:

$$
\begin{aligned}
& S_{N}=\left(t^{N}+\sum_{m \geq k} B_{N m}^{j} t^{m}\right)(d t)^{j} \quad-\infty<N \leq k-1 \\
& V_{M}=\left(t^{M}+\sum_{m \geq-k} B_{M m}^{1-j} t^{m}\right)(d t)^{1-j} \quad M \leq-(k+1)
\end{aligned}
$$

where $k=(2 j-1)(g-1)$. Moreover

$$
\oint S_{N} V_{M}=B_{N,-M-1}^{j}+B_{M,-N-1}^{1-j}=0
$$

Proof: From the Riemann-Roch theorem we know

$$
\begin{aligned}
\operatorname{dim} H^{\circ}\left(\omega^{j} \otimes O(N p)\right)-\operatorname{dim} H^{0}\left(\omega^{1-j} \otimes O(-N p)\right) & =\operatorname{dim} H^{\circ}\left(\omega^{j} \otimes O(N p)\right) \\
& =(2 j-1)(g-1)+N
\end{aligned}
$$

So for all $-\infty<N \leq k-1$ the $S_{N}$ exist with a zero of order $N$. On the other hand, applying Riemann-Roch with $j \rightarrow 1-j$ only gives

$$
\operatorname{dim} H^{0}\left(\omega^{1-j} \otimes O(N p)\right)=N-k
$$

for $N>2 j(g-1)$. Let $S_{0}, \ldots, S_{k-1}$ be a basis for $H^{0}\left(\omega^{j}\right)$. At $p$ we have, in a loca! coordinate $t, S_{i}=f_{i}(t)(d t)^{j}$, so if

$$
\operatorname{det}\left(\begin{array}{ccc}
f_{0} & \cdots & f_{k-1} \\
\cdot & \cdots & \cdot \\
f_{0}^{(k-1)} & \cdots & \cdot \\
f_{k-1}^{(k-1)}
\end{array}\right) \neq 0
$$

which is true for generic points, then we can choose our basis according to the theorem. Now choose a covering $U, \Sigma-p$ for the surface. Then the Serre duality isomorphism

$$
H^{0}\left(\omega^{j}\right)^{*}=H^{1}\left(\omega^{1-j}\right)
$$


can be represented explicitly as follows. If $\xi \in \Gamma\left(\left.\omega^{1-j}\right|_{U \cap(\Sigma-p)}\right)$ we have $\mid \xi j \rightarrow L_{\xi}$ where

$$
L_{\xi}: S \rightarrow \oint S \xi
$$

Therefore we may choose a basis

$$
t^{-1}(d t)^{1-j}, \ldots, t^{-k}(d t)^{1-j}
$$

Suppose we have a meromorphic section of $\omega^{1-j}$ which is holomorphic off of $p$, then its restriction to $U \cap(\Sigma-p)$ must define the zero cohomology class, so there are gaps at the first $\mathrm{k}$ poles. This shows the existence of $v_{M}$ as given in the theorem, at least at generic points.

Finally, since $S_{N}, V_{M}$ are holomorphic on $\Sigma-p$ we may slip the contour off the back of the surface, giving the last equality.

Regarding this theorem we should make two remarks. First, for the ordinary Weierstrass theorem we have $j=1$. Just change $k$ to $g$. Second, the determinant is a section of $\omega^{M}$ where

$$
\begin{array}{rlrl}
M & =\frac{1}{2}(2 j-1)^{2} g(g-1) & & j \geq 3 / 2 \\
& =\frac{1}{2} g(g+1) & j=1
\end{array}
$$

there are therefore $(2 g-2) M$ special points. 


\section{References}

(1) A. Beilinson, Y. Manin and Y. A. Shechtman : Localization of Virasoro and NeveuSchwarz Algebras preprint 1986; M. Kontsevich, Funct. Anal. and Appl. 21 (1987)156 [=Funk. Anal. Pril. 21(1987)78]

[2] A. Belavin, A. Polyakov and A. Zamalodchikov : Nucl. Phys. B241 (1984) 333.

(3] D. Friedan, E. Martinec and S. Shenker :Nucl. Phys. B271 (1986) 93.

(4) D. Friedan S. Shenker : Phys.Lett. 175B ( 1986) 287 ; Nucl. Phys. B281 (1987)509.

(5) S. Giddings and E. Martinec : Nucl. Phys. B278 (1986) 91 ;

E. Martinec, Nucl. Phys. B281 (1987) 157 ;

H. Sonoda, Berkeley preprint LBL-23260.

(6] S. Saito : Tokyo Metropolitan Univ. Preprints TMUP-HEL-8613/8615/8701/8707.

(7) N. Ishibashi, Y. Matsuo and H. Ooguri , Univ. of Tokyo Preprint UT-499 (1986).

[8] L. Alvarez-Gaume,C. Gomez and C. Reina : Phys. Lett. 190B (1986) 55.

(9] C. Vafa : Phys. Lett. 190B (1986) 47.

[10] L. Alvarez-Gaumé , C. Gomez and C. Reina : Proc. of the Trieste Spring School on Superstrings, CERN-TH 4775/87.

(11) E. Witten: "Quantum Field Theory, Grassmannians and Algebraic Curves ",PUPT1057 , May 1986 , to appear in C.M.P. .

[12] G. Segal : Talk presented at the conference "Links Between Geometry and Mathematical Physics", held at Schlöss Ringberg , March 1987 ; based on joint work with D. Quillen .

113] C. Vafa : "Conformal Theories and Punctured Riemann Surfaces" , HUTP-87/A066

[14] E. Arbarello, C. DeConcini, V. Kac, and C. Procesi, "Moduli Spaces of Curves and Representation theory", preprint.

(15) For details and references see for example L. Alvarez-Gaumé and P. Nelson : Lectures on Riemann Surfaces and String Theory ". Proc. of the.Trieste School on Superstrings 1986 . R. Iengo and E. Sezgin eds. World Scientific (1986). See also section 2 of [10!.

(16) For a thorough treatment of infinite grassmannians and references to earlier literature see G. Segal and G. Wilson : Publ. Math. de l'I.H.E.S. 61 (1985) 1 , and A. Pressley and G. Segal :Loop Groups ,Oxford U. Press , 1986 .

(17) See for example R. Gunning 'Lectures on Riemann Surfaces'. Princeton U. Press (1966).

(18] For details on prime forms and theta functions see for example: J. Fay : Theto Func. tions on Riemann Surfaces . Springer Verlag (1973).

(19) See for example L. Faddeev and A. Slavnov : Gauge Fields : The Quantum Theory. Benjamin Publisher (1981)

(20] L. Alvarez-Gaumé , J. B. Bost ,G. Moore P. Nelson and C. Vafa, Phys. Lett. 178B (1986) 41 , and Comm. Math. Phys. 112 (1987) 503. 
(21) E. Verlinde and H. Verlinde, Nucl. Phys. B288 (1987) 357.

[22] T. Eguchi and H. Ooguri, Phys.Lett. 187B (1987) 127.

[23] V. Knizhnik, Phys. Lett. 180B (1986) 247 , A. Redlich and H. Schnitzer and K. Tsokos, Nucl. Phys. B289 (1987) 397 , H. Schnitzer and K. Tsokos, Partition Function and Fermi Bose Equivalence for Simply-Laced Groups ; BRX TH-215 , M. Dugan and H. Sonoda, Nucl. Phys. B289 (1987) 227 , J. Bagger, D. Nemeschansky, N. Seiberg and S. Yankielowicz, Nucl. Phys. B289 (1987) 53.

[24] P. Di Vecchia, K. Hornfeck, and M.Yu, Nordita preprint-87/29 P.

[25] L. Ahlfors, Complex Analysis, Mc Graw Hill 1953 ;

J. Conway, Functions of one complex variable, Springer Verlag-1978.

[26] R. Marnelius : Nucl. Phys. B211 (1983) 14.

[27] Y. Matsuo UT-511, Univ. of Tokyo Preprint ; D. Espriu : IASSNS-HEP-87/35.

[28] K. Kodaira Complex Manifolds and Deformations of Complex Structures. Springer Verlag 1986.

[29] I. M. Gelfand and D.B. Fuks, Funk. Anal. Pril. 2(1968)92

[30] G. Segal, Comm. Math. Phys. 80(1981)301

(31] Schiffer and Spencer :Functionals on Finite Riemann Surfaces, Princeton University Press 1954.

[32] See for example D. Friedan, "Introduction to Polyakov's string theory," in Recent advances in field theory and statistical mechanics J.-B. Zuber and R. Stora, eds. Les Houches 1982 Amsterdam: Elsevier 1984

[33] H. Masur : Duke Math. J. 43 (1976) 623.

[34] L. Bers, Bull. of Am. Math. Soc. Vol 5 (1981) 131.

[35] A. LeClair , M.E. Peskin and C.R. Preitshopf, to appear ;

A. Neveu and P. West, CERN-TH.4757/87;

P. Di Vecchia, R. Nakayama, J.L. Peterson, J. Sidenius, and S. Sciuto, Phys. Lett.182B (1986) 164.

(36] A. LeClais, HUTP-87/A033 .

(37) J. Harer Inv. Math. 72 (1983) 221.

[38] A. Belavin,V. Knizhnik : Phys. Lett. B168 (1986) 201.

[39] A. M. Polyakov : Phys. Lett. B103 (1981) 211 ; D. Friedan in Les Housches 1982 : Recent Advances in Field Theory on Statistical Mechanics . J.B. Zuber and R. Stora Eds. North Holland, 1984 . O. Alvarez : Nucl. Phys. B216 (1983) 125 ; G. Moore and P. Nelson : Nucl. Phys. B266 (1986) 58 ; E. D'Hoker and D.H. Phong Nucl Phys. B269 (1986) 205 .

[40] I. Frenkel, H. Garland and G. Zuckermann, Proc. Nat. Acad. Sci., Vol. 83 (1986) 8442. 
[41] See for example [3] and M. Green, J. Schwarz and E. Witten, String Theory. Cambridge U.P., 1987 for thorough reference to the literature .

[42] See, e.g. M.D. Freeman and D.I. Olive, Phys. Lett. 175B (1986)151.

[43] A.B. Zamolodchikov and V.A. Fateev. Zh. Eksp. Theor. Fiz. 90 (1986) 1553;

L. Dixon, J. Harvey , C. Vafa and E. Witten, Nucl. Phys. B261 (1985) 651 ; B274 (1986) 285 ;

D. Gepner and E. Witten, Nucl. Phys. B278 (1986) 493 ;

H. Kawai, D. Lewellen and H. Tye, CLNS $86 / 751$;

K.S. Narain, M.H. Sarmadi, and C. Vafa, Nucl. Phys. B279 (1987) 369

[44] P. Ginsparg, Harvard preprint, HUTP-87/A068;

D. Gepner, Princeton preprint, 1987. ;

D. Kastor, E. Martinec, and Z. Qiu, Chicago preprint EFI-87-58;

J. Bagger, D. Nemeschansky, and S. Yankielowicz, Harvard preprint HUTP-87/A073 ;

F.A. Bais, P. Bouwknegt, M. Surridge, K. Schoutens, ITFA 87-18.

[45] I.G. Koh, and H.J. Shin, KAIST-87/6

[46] R. Dijkgraaf, E. Verlinde and H. Verlinde, Utrecht preprint THUj-87/17.

[47] S. Hamidi, and C. Vafa, Nucl. Phys. B279 (1987) 465;

[48] C. Vafa, unpublished.

[49] E. Corrigan, DTP-87/19;

J.H. Schwarz and C.C. Wu, Nucl. Phys. B72 (1974) 397 ;

E. Corrigan and D.B. Fairlie, Nucl. Phys. B91 (1975) 527 ;

M.B. Green, Nucl. Phys. B103 (1976) 333 ;

J.H. Schwarz, Phys. Rep. 89 (1982) 223.

[50] D. Friedan, Z. Qiu and S. Shenker, Phys. Rev. Lett. 52 (1984) 1575

[51] V. Dotsenko, and V. Fateev, Nucl. Phys. B240 (1984) 312; Nucl.Phys.B251 (1985) 691.

[52] H. Sonoda, Nucl. Phys. B281 (1987) 546;

T. Eguchi and H. Ooguri, Nucl. Phys. B282 (1987) 308.

(53) T. Eguchi and H.Ooguri, University of Tokyo preprint UT.518.

[54] D. Kazhdan and C. Vafa, unpublished.

[55] D. Bernard, "On the Wess-Zumino-Witten Models On The Torus," princeton preprint, PUPT-1076

(56) For details and references to the present state of affairs with the measure in supermoduli space, see : J. Atick, G. Moore and A. Sen, IASSNS-HEP-87/61, and H. Verlinde Utrecht preprint THU-87/26.

[57] Work in progress. 
[58] V.G. Knizhnik, Comm. Math. Phys. 112(1987)567

[59] See for example B. Simon : Adv. in Math. 24 (1977) 244.

[60] B. A. Dubrovin, I. M. Krichever and S. P. Novikov : Sov. Scien. Reviews 3 (1982) 1 ; and I. M. Krichever : Russ. Math. Surveys 32 (1977) 185

[61] I.B. Frenkel and V. G. Kac : Invent. Math. 62 (1980) 23.

$62]$ G. Segal : Comm. Math. Phys. 80 (1981) 301.

[63] V.G. Kac and D. H. Peterson : I.H.E.S./M/85/63. See also V. G. Kac :Infinite Dimensional Algebras. Birkhauser, Boston (1983) .

[64] S. Mandelstam, Phys. Rev. D11 (1975) 3026.

[65] T. Banks et. al., Nucl. Phys. B108 (1976) 119.

[66] E. Date, M. Jimbo, M. Kashiwara, and T. Miwa : J. of Phys. Soc. of Japan 50 (1981) 3806 .

[67] H. Farkas and I. Kra :Riemann Surfaces. Springer Verlag (1980). 


\section{Figure Captions}

Fig. 2.1: We associate the state $|B(W)\rangle$ to the boundary of the disc. The state $\langle 0|$ is associated with the puncture (the infinite past). The fields are inserted at the points inside the disc $(z$ and $w$ ).

Fig. 2.2: A Riemann surface $\Sigma$ with a puncture (point) $p$ and a coordinate $z$ defined in a neighborhood of the puncture.

Fig. 2.3: A Riemann surface $\Sigma$ is divided into two parts by the circle $S^{1}$.

Fig. 2.4: A Riemann surface $\Sigma$ with $n$ punctures $p_{i}$ and $n$ coordinates in the neighborhood of punctures.

Fig. 2.5: A Riemann surface $P$ joins another one $Q$ at the punctures $i$ and $j$, to give a new surface $R$. Similarly, two coordinate patches of a single Riemann surface $P$ can be identified to give a new surface $P 8_{j}^{i}$.

Fig. 4.1: The Riemann surface $\Sigma$ with puncture $p$ and coordinate in the neighborhood of puncture, can be deformed by cutting out a disc $D$, deforming the annulus from $A$ to $A^{\prime}$ and then gluing it back to the surface (for example the point $q^{\prime}$ is identified with the point $q$ ). 


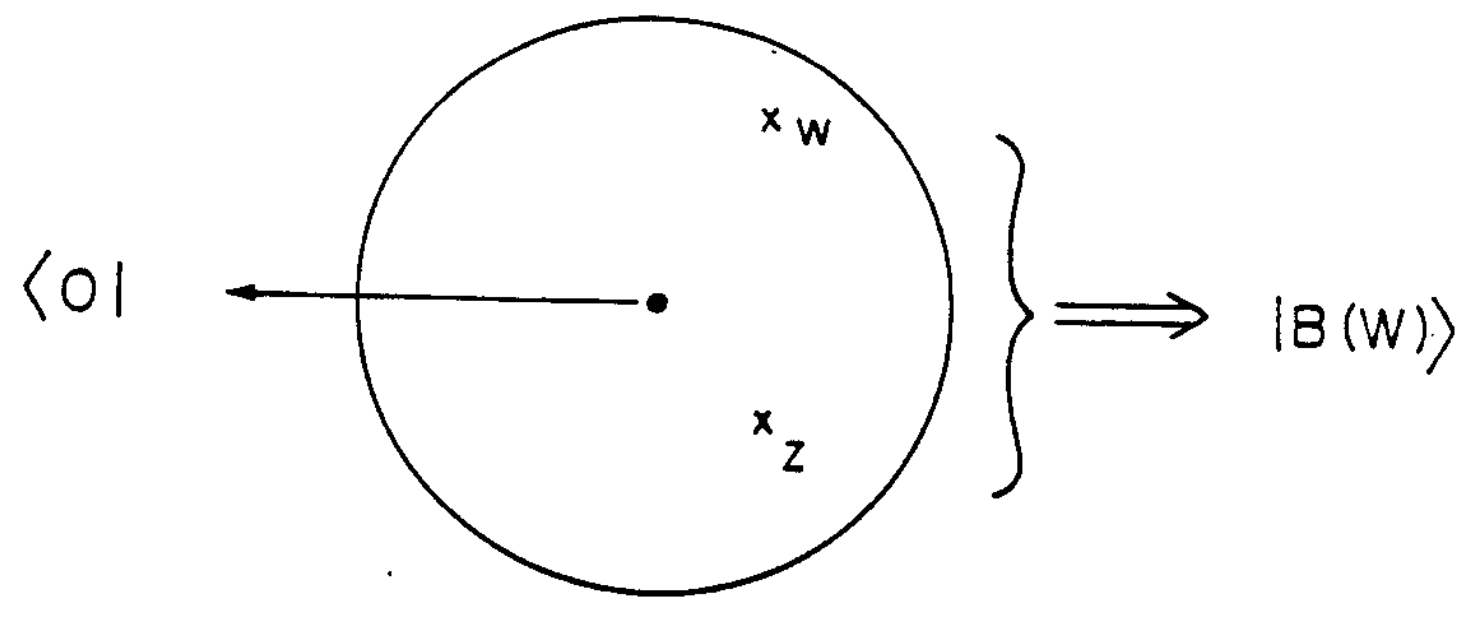

Figure 2.1

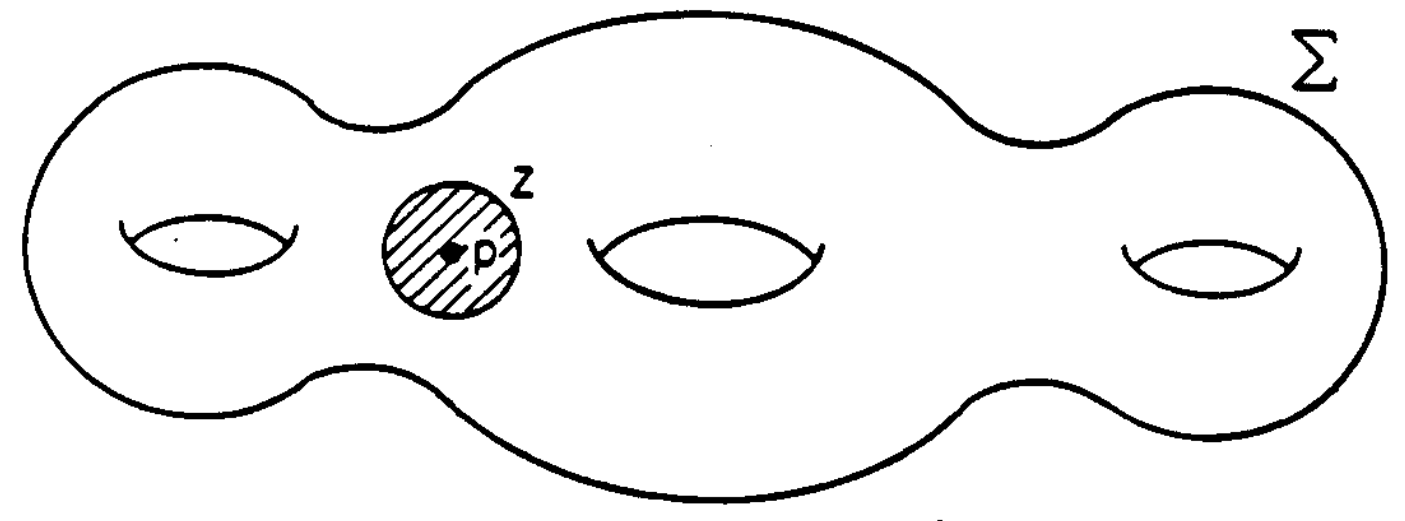

Figure 2.2 


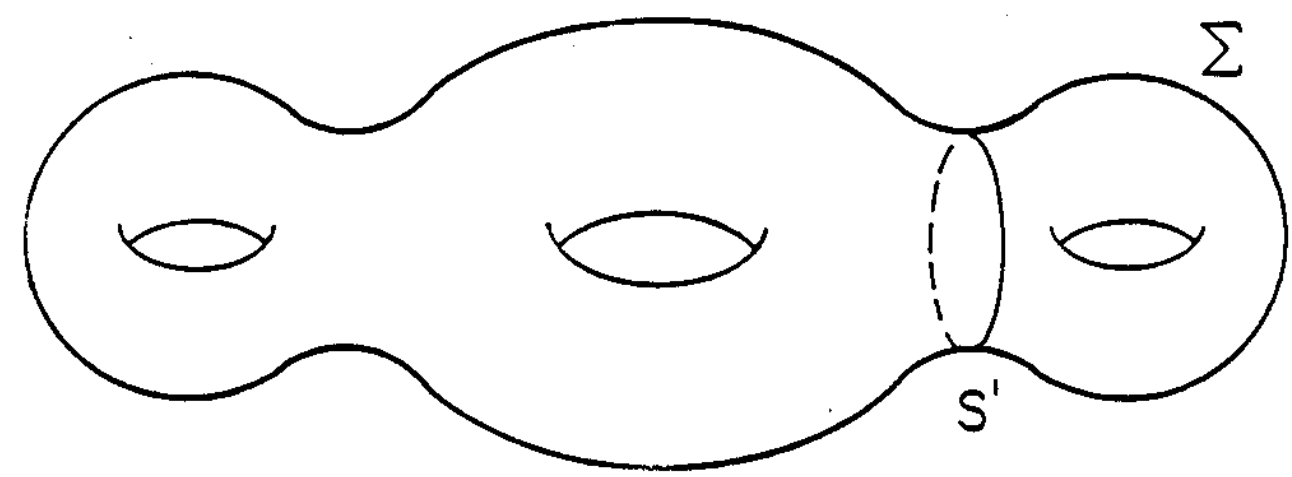

Figure 2.3

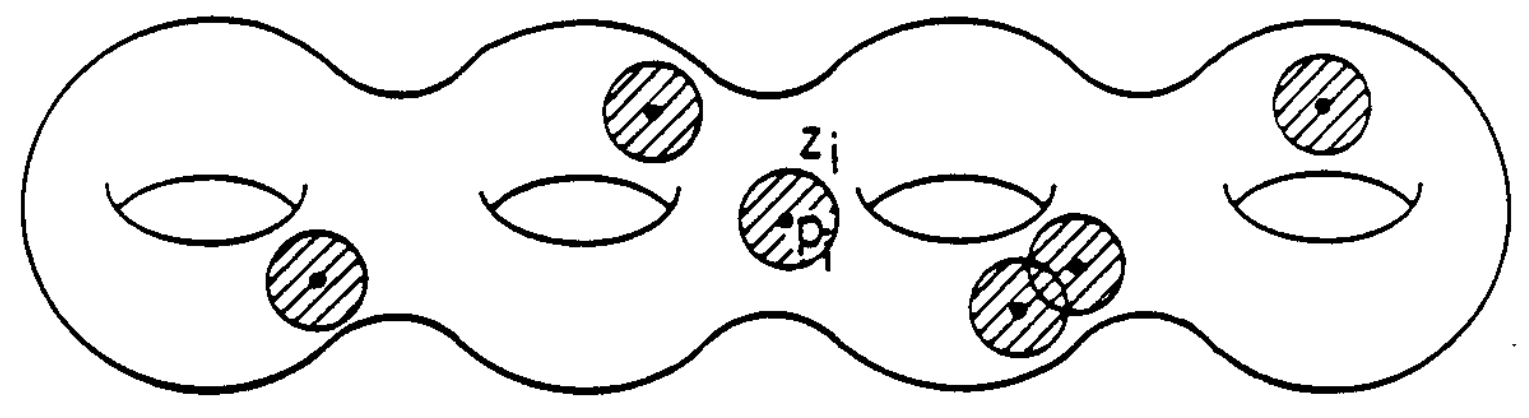

Elgure 2.4 


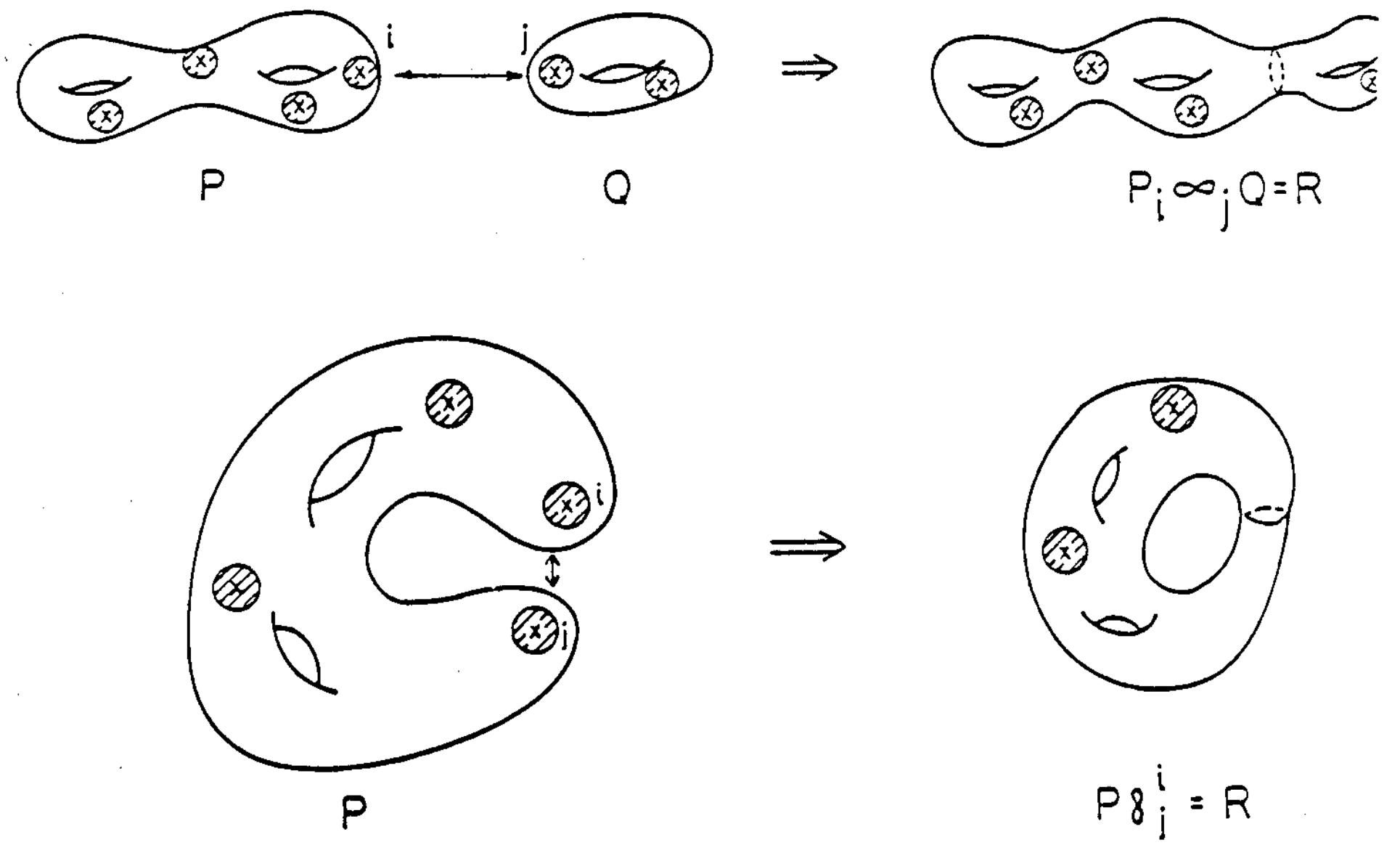

Figure 2.5 


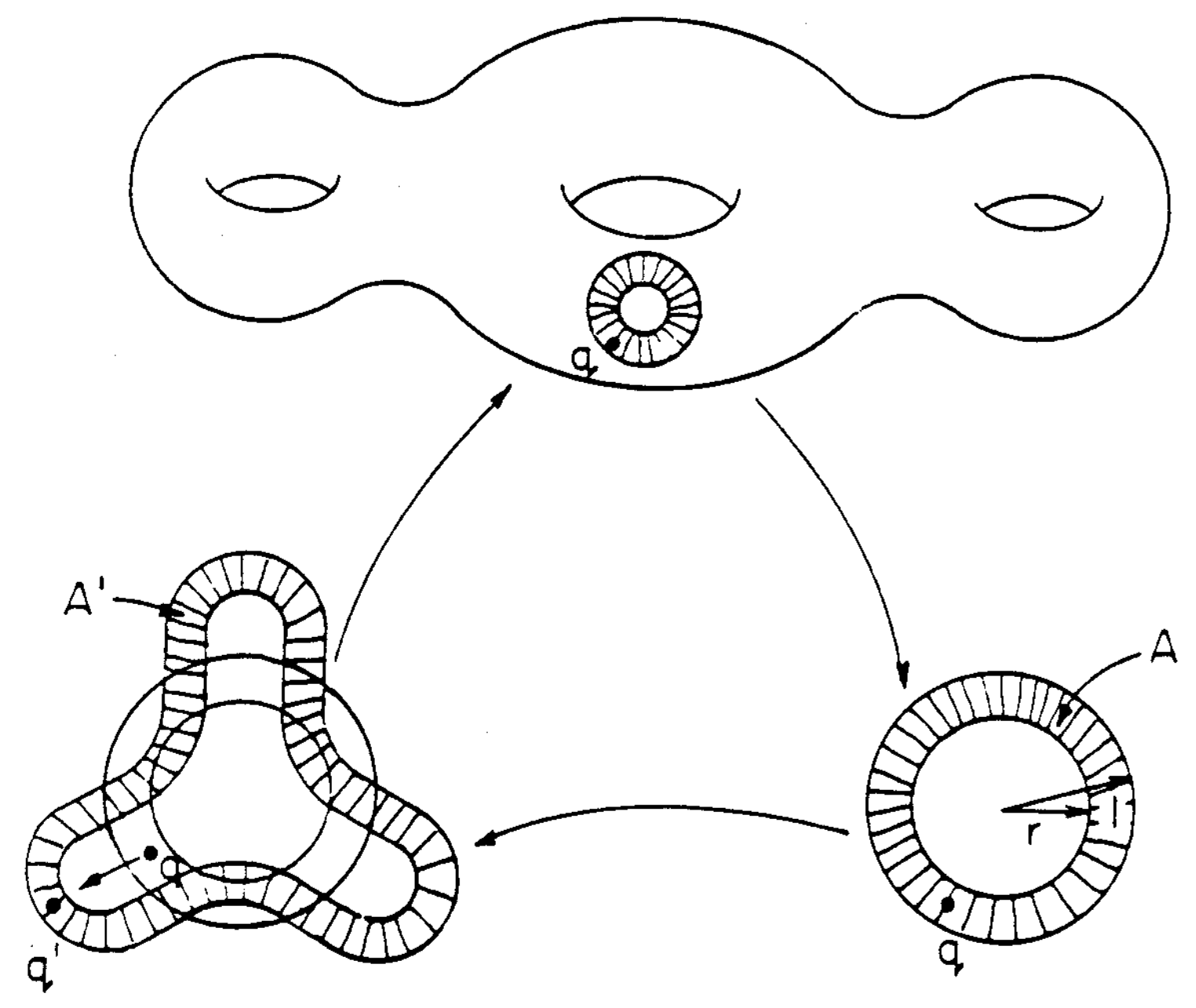

Figure 4.1 